\title{
Diffusive limit for finite velocity Boltzmann kinetic models
}

\section{Pierre Louis Lions and Giuseppe Toscani}

\begin{abstract}
We investigate, in the diffusive scaling, the limit to the macroscopic description of finite-velocity Boltzmann kinetic models, where the rate coefficient in front of the collision operator is assumed to be dependent of the mass density. It is shown that in the limit the flux vanishes, while the evolution of the mass density is governed by a nonlinear parabolic equation of porous medium type. In the last part of the paper we show that our method adapts to prove the so-called Rosseland approximation in radiative transfer theory.
\end{abstract}

\section{Introduction.}

In the kinetic theory of rarefied gases, two-velocity models of the Boltzmann equation are supposed to describe the evolution of the velocity distribution of a fictitious gas composed of two kinds of particles that move parallel to the $x$-axis with constant and equal speeds, either in the positive $x$-direction with a density $u$, or in the negative $x$-direction with a density $v$. The most general two-speed gas which is in local equilibrium when $u=v$ is described by the equations

$$
\left\{\begin{array}{l}
\frac{\partial u}{\partial t}+c \frac{\partial u}{\partial x}=k(u, v, x)(v-u), \\
\frac{\partial v}{\partial t}-c \frac{\partial v}{\partial x}=k(u, v, x)(u-v), \quad x \in \mathbb{R}, t \geq 0
\end{array}\right.
$$


where $c$ is the modulus of the constant speed of the particles, and $k$ is a nonnegative rate coefficient.

The most famous example of these models was proposed by Carleman's in the 1930's and appeared in print for the first time in 1957 in [Car]. In Carleman's model $k(u, v, x)=u+v$, so that the "collision" terms on the right-hand side of (1.1) describe binary interactions between particles. An interaction between two molecules of the former type results into two molecules of the latter type and vice versa. Clearly, Carleman equations have no meaningful physical interpretation; in particular, there is no conservation of momentum.

Choosing $k(u, v, x)=1$, we obtain a linear system, known as Goldstein-Taylor model [Gol], [Tay]. The system represents the forward equation for the density of a molecule moving with constant speed along the $x$-axis, subject to spontaneous reversals of directions, at the jump times of a standard Poisson process of unit rate.

The macroscopic variables for these models are the mass density $\rho=u+v$, and the flux $j=c(u-v)$. It is interesting to remark that, since $u$ and $v$ can be expressed in terms of $\rho$ and $j$, so that $k(u, v, x)=k(\rho, j, x)$, system (1.1) is equivalent to the following macroscopic equations for the mass density and the flux

$$
\left\{\begin{array}{l}
\frac{\partial \rho}{\partial t}+\frac{\partial j}{\partial x}=0 \\
\frac{\partial j}{\partial t}+c^{2} \frac{\partial \rho}{\partial x}=-2 k(\rho, j, x) j, \quad x \in \mathbb{R}, t \geq 0,
\end{array}\right.
$$

Basically, two different types of problems for the system (1.1) can be formulated. The first one is the initial or initial-boundary value problem. The second one is an asymptotic problem. Let us assume that the mean free path is not normalized to unity, but is left in the equation as a "small" parameter $\varepsilon$. More precisely, this means that in (1.1) we replace $k$ by $k / \varepsilon$. The following question then naturally arises: what is the limiting form of system (1.2) as $\varepsilon \rightarrow 0$, and how do the initial data of the limiting equation match the initial data associated with (1.2)?

The limit $\varepsilon \longrightarrow 0$ corresponds to the transition from a kinetic description of the gas to that of a gas as a continuum, and we refer to the asymptotic problem as the hydrodynamic limit associated with the kinetic system (1.1).

Much is known for Carleman's equation in the scaling

$$
\frac{\partial u}{\partial t}+\frac{1}{\varepsilon} \frac{\partial u}{\partial x}=\frac{1}{\varepsilon^{2}}\left(v^{2}-u^{2}\right)
$$




$$
\frac{\partial v}{\partial t}-\frac{1}{\varepsilon} \frac{\partial v}{\partial x}=\frac{1}{\varepsilon^{2}}\left(u^{2}-v^{2}\right)
$$

This asymptotic problem was first investigated by Kurtz [Kur]. By means of the theory of nonlinear semigroups, he proved that, for initial data $u_{0}(x)=v_{0}(x) \in L^{1}(\mathbb{R})$ the mass density $\rho_{\varepsilon}(x, t)$ converges in $L_{x}^{1}$ for all $t \geq 0$ to $\rho(x, t)$ satisfying the nonlinear diffusion equation

$$
\frac{\partial \rho}{\partial t}=\frac{1}{4} \frac{\partial}{\partial x}\left(\frac{1}{\rho} \frac{\partial \rho}{\partial x}\right), \quad x \in \mathbb{R}, t \geq 0,
$$

while $j_{\varepsilon}(x, t)$ converges to zero. In other words, (1.4) is the hydrodynamical limit of the Carleman's equation (1.3).

Subsequently, McKean [McK] generalized the preceding result, by removing the restriction that the initial flux has to be taken equal to zero.

Further results are due to Kaper, Leaf and Reich [KLR], who investigated the problem treated by Kurtz with $\varepsilon$-dependent initial data, and to Fitzgibbon [Fi1], [Fi2] who studied the problem in a bounded domain with specular reflecting boundary conditions. The method of proof of all the aforementioned papers relies mainly on the theory of nonlinear semigroups, and on the fact that these problems are $L^{1}$-accretive.

McKean's result $[\mathrm{McK}]$, has been recently extended by Toscani and Pulvirenti [PTo] to the system

$$
\left\{\begin{array}{l}
\frac{\partial u}{\partial t}+\frac{1}{\varepsilon} \frac{\partial u}{\partial x}=\frac{1}{\varepsilon^{2}} \rho^{\alpha}(v-u) \\
\frac{\partial v}{\partial t}-\frac{1}{\varepsilon} \frac{\partial v}{\partial x}=\frac{1}{\varepsilon^{2}} \rho^{\alpha}(u-v)
\end{array}\right.
$$

with $0 \leq \alpha \leq 1$. The system (1.5) includes as particular cases both Carleman's equation $(\alpha=1)$ and the Golstein-Taylor model $(\alpha=0)$.

In the present paper, we will investigate in the diffusive limit the system

$$
\left\{\begin{array}{l}
\frac{\partial \rho_{\varepsilon}}{\partial t}+\frac{\partial j_{\varepsilon}}{\partial x}=0, \\
\varepsilon^{2} \frac{\partial j_{\varepsilon}}{\partial t}+\frac{\partial \rho_{\varepsilon}}{\partial x}=-2 \rho_{\varepsilon}^{\alpha} j_{\varepsilon}, \quad x \in \mathbb{R}, t \geq 0 .
\end{array}\right.
$$

for any value of $\alpha<1$. The case $\alpha<-1$ is of particular interest since we obtain in the limit the well-known porous media equation

$$
\frac{\partial \rho}{\partial t}=\frac{1}{2(1+|\alpha|)} \frac{\partial^{2}}{\partial x^{2}} \rho^{1+|\alpha|} .
$$


In the second part of the paper we will investigate the multidimensional and in particular the three dimensional version of system (1.1). In three dimensions of space, the molecules of the fictitious gas can move in directions parallel to one of the axes $x_{1}, x_{2}, x_{3}$ either in the positive direction or in the negative direction. Denoting by $u_{i}$ (respectively $\left.u_{i+3}\right), i=1,2,3$ the densities of molecules moving in the positive (respectively negative) $x_{i}$-directions, the most general system which is in local equilibrium when $u_{i}=\rho / 6, i=1,2, \ldots, 6$, where $\rho=\sum u_{i}$ is the mass density, takes the form

$$
\left\{\begin{array}{l}
\frac{\partial u_{i}}{\partial t}+c \frac{\partial u_{i}}{\partial x_{i}}=k\left(u_{1}, \ldots, u_{6}, x\right)\left(\rho-6 u_{i}\right), \\
\frac{\partial u_{i+3}}{\partial t}-c \frac{\partial u_{i+3}}{\partial x_{i}}=k\left(u_{1}, \ldots, u_{6}, x\right)\left(\rho-6 u_{i+3}\right),
\end{array}\right.
$$

with $i=1,2,3, x \in \mathbb{R}^{3}$, and $t \geq 0$. With a few modification, our one-dimensional analysis extends to the three-dimensional case when $k\left(u_{1}, \ldots, u_{6}, x\right)=\rho^{\alpha}, \alpha<1$.

Other models (one or multidimensional) can be studied with our technique. Among them, let us mention the cases $k(u, v, x)=a(x)$, and $k(u, v, x)=\sum_{l=1}^{m} u^{m-l} v^{l}, m \in \mathbb{N}^{+}$.

The main object of the present investigation is to justify the passage from the mesoscopic description of kinetic theory to the macroscopic one of continuum theory. This passage is usually described by the asymptotic relations between solutions of the Boltzmann equation and solutions of Euler and Navier-Stokes equations. It is worthwhile mentioning that the target equations of continuum theory can be obtained directly from a microscopic description. In particular, the deduction of diffusion equations as a hydrodinamic limit of particle model is a well-studied subject. We quote here the paper by K. Oelschläger [Oel], in which the porous medium equation is obtained as a limit of a particle system that interact under the action of adequate potentials, as the number of particles tends to infinity. Depending on the scaling parameter applied, different versions of the porous medium equation in the limit dynamics are obtained. A different aspect of the limit dynamics for a Markov system of many particles, and the convergence to the porous media equation of the empirical density of the number of particles has been investigate by Inoue [Ino]. In this paper, the Kac-McKean propagation of chaos for the system is shown to hold.

In Section 2 we discuss the initial and the initial-boundary value problems associated with (1.1), and we will recover elementary a priori 
estimates for the solution. Here, the models (1.5) naturally separate in two subclasses, corresponding to $|\alpha| \leq 1$ and $\alpha<-1$ respectively. When $|\alpha|<1$, at least in one dimension, the problem is shown to be $L^{1}$-accretive. Entropy bounds are discussed in Section 3, and the limit theorems in Section 4. When the model is accretive, given initial values of bounded variation, $L^{1}$-contraction and translational invariance imply total variation bounds on the solution, and one can pass to the limit for general $L^{1}$ initial conditions, in a rather straightforward (and standard) way. Let us briefly discuss the case $\alpha>0$. The entropy bounds of Section 3, Theorem 3.1, imply that $\left\{j_{\varepsilon}\right\}$ is bounded in $L^{2}$, and thus by the second of equations (1.6) $\partial \rho_{\varepsilon} / \partial t$ is bounded in $L^{2}\left(0, T ; H_{\mathrm{loc}}^{-1}\right)$ for all $T>0$. In view of the a priori bounds of Section $2,\left\{\rho_{\varepsilon}\right\}$ is bounded in $L^{\infty}(\mathbb{R})$.

These bounds, combined with Proposition 2.3, imply that the family $\left\{\rho_{\varepsilon}\right\}$ is relatively compact in $C\left([0, T] ; L^{1}(\mathbb{R})\right)$ for all $T>0$.

Hence $\rho_{\varepsilon}^{\alpha} j_{\varepsilon} \longrightarrow \rho^{\alpha} j$ in $L^{2}$-weak, $\varepsilon^{2} j_{\varepsilon} \longrightarrow 0$ strongly in $L^{2}$ and from the flux equation we deduce

$$
\frac{\partial \rho}{\partial x}=-2 \rho^{\alpha} j
$$

at least in the sense of distributions (and in fact in $L^{2}$ ). Considering that $\rho \in L^{\infty}$, the above equality implies that we have

$$
j=-\frac{1}{2(1-\alpha)} \frac{\partial \rho^{1-\alpha}}{\partial x}
$$

in $L^{2}$. The case $-1 \leq \alpha<0$ follows with similar arguments.

The case $\alpha \leq-1$ is more delicate, and the result is achieved by compensated compactness theory (see F. Murat [Mu1], [Mu2], and L. Tartar [Ta1], [Ta2]). In Section 5, we extend our analysis to the threedimensional models (1.8). Finally, we state without proofs various extensions and variants of the results obtained below. In fact, our method of proof adapts to models with a continuous set of velocities. In particular we make contact (and propose more general proofs) with the so-called Rosseland approximation in radiative transfer theory (see C. Bardos, F. Golse, B. Perthame and R. Sentis [BGPS], and the references therein). 


\section{Basic a priori estimates and global existence.}

In this section we discuss the initial and the initial-boundary value problems for system (1.1). Many arguments that follow are very elementary, and the proofs will be omitted. Besides, it has to be pointed out that the general a priori estimates we will use in the sequel, to our knowledge has never been used before.

For our purposes, as will be clear later on, we need to study (1.1) in a bounded interval $\Omega=(-a, a)$ with periodic boundary conditions. This limitation allows us to prove existence and uniqueness of a solution under weak conditions on the rate coefficient $k$.

Useful a priori estimates for the solution to system (1.1) follow by the structure of the "collision" term. Taking the sum of the two equations, and integrating over $\Omega$, we obtain the mass conservation, namely $\int_{\Omega} \rho(x, t) d x$ is independent of $t \geq 0$. Let now $\varphi(r), r \geq 0$ be a (regular) convex function. If we multiply the first equation of system (1.1) by $\varphi^{\prime}(u)$ and the second by $\varphi^{\prime}(v)$, after integrating over $\Omega$ we obtain

$$
\begin{aligned}
\int_{\Omega} \frac{\partial \varphi(u)}{\partial t} & +\int_{\Omega} \frac{\partial \varphi(v)}{\partial t} \\
& =-\int_{\Omega} k(u, v, x)(u-v)\left(\varphi^{\prime}(u)-\varphi^{\prime}(v)\right) d x
\end{aligned}
$$

Since $\varphi^{\prime}(r)$ is non decreasing, the right-hand side of (2.1) is non positive. Thus we deduce, at least formally, that $\int_{\Omega}(\varphi(u)+\varphi(v)) d x$ is monotone non increasing in $t \geq 0$, and

$$
\begin{aligned}
\int_{\Omega}(\varphi(u(x, t))+\varphi( & v(x, t))) d x \\
& \leq \int_{\Omega}\left(\varphi\left(u_{0}(x)\right)+\varphi\left(v_{0}(x)\right)\right) d x .
\end{aligned}
$$

In particular, if the initial densities $u_{0}, v_{0}$ belong to $L^{\infty}(\Omega)$, taking $\varphi(r)=r^{p}$ for any $p \geq 1$, and letting $p$ go to $+\infty$, we deduce the following bound

$$
\max \left\{\|u(t)\|_{\infty},\|v(t)\|_{\infty}\right\} \leq \max \left\{\left\|u_{0}\right\|_{\infty},\left\|v_{0}\right\|_{\infty}\right\} .
$$

Similarly, we may assume that $u_{0}(x) \geq \delta, v_{0}(x) \geq \delta$ in $\Omega$ for some $\delta \geq 0$. Then, choosing $\varphi(r)=r^{-p},(p>0)$ in (2.2) above, and letting 
$p$ go to $+\infty$ we obtain

$$
\max \left\{\left\|\frac{1}{u(t)}\right\|_{\infty},\left\|\frac{1}{v(t)}\right\|_{\infty}\right\} \leq \max \left\{\left\|\frac{1}{u_{0}}\right\|_{\infty},\left\|\frac{1}{v_{0}}\right\|_{\infty}\right\},
$$

or, equivalently, for all $t \geq 0$

$$
\inf _{x \in \Omega}\{u(x, t), v(x, t)\} \geq \inf _{x \in \Omega}\left\{u_{0}(x), v_{0}(x)\right\} .
$$

These formal a priori estimates are sufficient to yield the global existence of a unique solution of system (1.1) for a large class of rate coefficients. More precisely, we now need to specify our basic assumptions on $k(u, v, x)$.

Definition 2.1. 1) $k(u, v, x)$ is an admissible rate coefficient of type 1 for system (1.1) if

a) $k(u, v, x) \leq c_{1}(\mu)<\infty$, if $u, v \leq \mu$ for any $\mu>0$, and $x \in \Omega$.

2) $k(u, v, x)$ is an admissible rate coefficient of type 2 if $k(0,0, x)=$ $\infty$ and

b) $k(u, v, x) \leq c_{2}(\lambda)<\infty$, if $u, v \geq \lambda>0$ for any $\lambda>0$.

A simple example is given by the rate coefficient

$$
k(u, v, x)=(u+v)^{\alpha} .
$$

$k$ is of type 1 if $\alpha \geq 0$, and of type 2 if $\alpha<0$.

We then have the following

Proposition 2.1. Let $0 \leq u_{0}(x), v_{0}(x) \in L^{\infty}(\Omega)$. Then, the initialboundary value problem for the system (1.1) with a rate coefficient of type 1 has a unique solution $u(x, t), v(x, t) \in L^{\infty}(\Omega \times(0, T)) \cap$ $C\left([0, T] ; L^{p}(\Omega)\right)$ for all $T>0,1 \leq p<\infty$. In addition, the solution satisfies the bound (2.3).

Proposition 2.2. Let $0 \leq u_{0}(x), v_{0}(x) \in L^{\infty}(\Omega)$ satisfy $u_{0}, v_{0} \geq \delta$ on $\Omega$ for some $\delta>0$. Then, the initial-boundary value problem for the system (1.1) with a rate coefficient type 2 has a unique solution bounded away from zero $u(x, t), v(x, t) \in L^{\infty}(\Omega \times(0, T)) \cap C\left([0, T] ; L^{p}(\Omega)\right)$ for all $T>0,1 \leq p<\infty$. In addition, this solution satisfies the bounds (2.3) and (2.4). 
A particular choice of $k$ obviously allows to obtain additional results for the initial-boundary value problem (1.1). As precised in the introduction, we are interested in the fluid-dynamical limit of system (1.5), that corresponds to the choice $k(u, v, x)=(u+v)^{\alpha}=\rho^{\alpha}$, where $\alpha \leq 1$ is a fixed constant. Since $(u+v)^{\alpha}$ is admissible, existence and uniqueness of a solution in $L^{\infty}$ follows by Proposition 2.1 when $\alpha$ is positive, or by Proposition 2.2 when $\alpha$ is negative.

We are now going to use a few simple facts from the theory of dissipative operators. Let $f=(u, v)$, and let $A_{\alpha}$ be the operator defined by

$$
A_{\alpha} f=\left(\rho^{\alpha}(v-u), \rho^{\alpha}(u-v)\right) .
$$

Then we have

Lemma 2.1. Let $0 \leq \alpha \leq 1$. Then, the operator $A_{\alpha}$ is dissipative from the domain

$$
D^{+}\left(A_{\alpha}\right)=\left\{(u, v) \in L^{1}(\Omega) \times L^{1}(\Omega),\|u\|_{\infty},\|v\|_{\infty}<\infty\right\}
$$

into $L^{1}(\Omega) \times L^{1}(\Omega)$.

If $-1 \leq \alpha<0$, and if $\delta>0$, the operator $A_{\alpha}$ is dissipative from the domain

$$
D_{\delta}^{+}\left(A_{\alpha}\right)=\left\{(u, v) \in L^{1}(\Omega) \times L^{1}(\Omega), u, v \geq \delta,\|u\|_{\infty},\|v\|_{\infty}<\infty\right\}
$$

into $L^{1}(\Omega) \times L^{1}(\Omega)$.

Proof. Let us recall that a closed operator $A$ from the domain $D(A) \subset$ $L^{+}(X)$ into $L^{1}(X)$ is dissipative if, for any functions $f_{1}, f_{2} \in D(A)$

$$
\int_{X}\left(A f_{1}-A f_{2}\right) \operatorname{sign}\left(f_{1}-f_{2}\right) d x \leq 0 .
$$

In our case, $f=(u, v)$, so that

$$
\begin{aligned}
\left(A_{\alpha} f_{1}-\right. & \left.A_{\alpha} f_{2}\right) \operatorname{sign}\left(f_{1}-f_{2}\right) \\
= & \left(\left(u_{1}+v_{1}\right)^{\alpha}\left(v_{1}-u_{1}\right)-\left(u_{2}+v_{2}\right)^{\alpha}\left(v_{2}-u_{2}\right)\right) \operatorname{sign}\left(u_{1}-u_{2}\right) \\
& +\left(\left(u_{1}+v_{1}\right)^{\alpha}\left(u_{1}-v_{1}\right)-\left(u_{2}+v_{2}\right)^{\alpha}\left(u_{2}-v_{2}\right)\right) \operatorname{sign}\left(v_{1}-v_{2}\right) .
\end{aligned}
$$

Then, the conclusion of the lemma follows by observing that, for $a \geq 0$, the function

$$
y=(x+a)^{\alpha}(x-a) .
$$


is monotone non decreasing for any fixed $\alpha \in[-1,1]$.

Let us now set

$$
B_{\alpha} f=\left(-\frac{\partial u}{\partial x}+\rho^{\alpha}(v-u), \frac{\partial v}{\partial x}+\rho^{\alpha}(u-v)\right)=\frac{\partial}{\partial x}(-u, v)+A_{\alpha} f
$$

Then, the following lemma is immediate

Lemma 2.2. Let $0 \leq \alpha \leq 1$. Then, the operator $B_{\alpha}$ is dissipative from the domain

$$
D^{+}\left(B_{\alpha}\right)=\left\{(u, v) \in W^{1,1}(\Omega) \times W^{1,1}(\Omega)\right\}
$$

into $L^{1}(\Omega) \times L^{1}(\Omega)$.

If $-1 \leq \alpha<0$, and if $\delta>0$, the operator $B_{\alpha}$ is dissipative from the domain

$$
D_{\delta}^{+}\left(B_{\alpha}\right)=\left\{(u, v) \in W^{1,1}(\Omega) \times W^{1,1}(\Omega), u, v \geq \delta\right\}
$$

into $L^{1}(\Omega) \times L^{1}(\Omega)$.

REMARK 2.1. If $0 \leq \alpha \leq 1$, the existence theory in $L^{\infty}$ can be extended to all of $\mathbb{R}$ without any difficulty. A further consequence of Proposition 2.1, combined with the a priori estimate (2.2) is that, if the initial data $u_{0}(x), v_{0}(x) \in L^{\infty}(\mathbb{R}) \cap L^{p}(\mathbb{R})$ for some $p \geq 1$, the solution $u(x, t), v(x, t) \in L^{\infty}(\mathbb{R}) \cap L^{p}(\mathbb{R})$ and

$$
\left(\int_{\mathbb{R}}\left(u(x, t)^{p}+v(x, t)^{p}\right) d x\right)^{1 / p}
$$

is monotone non increasing for $t \geq 0$.

By Lemma 2.2, provided the initial values are in $D^{+}\left(B_{\alpha}\right)$, the solution of the system (1.5) can be written as

$$
(u(\cdot, t), v(\cdot, t))=e^{t B_{\alpha}}\left(u_{0}(\cdot), v_{0}(\cdot)\right)
$$

and, given $f_{1}=\left(u_{1}, v_{1}\right), f_{2}=\left(u_{2}, v_{2}\right)$

$$
\left\|e^{t B_{\alpha}} f_{1}-e^{t B_{\alpha}} f_{2}\right\|_{1} \leq\left\|f_{1}-f_{2}\right\|_{1} .
$$


In particular, if the initial densities $\left(u_{0}(x), v_{0}(x)\right)$ are of bounded variation, we see that the solution $(u(x, t), v(x, t))$ is of bounded variation, and

$$
\begin{aligned}
\max \left\{\left\|\frac{\partial u(x, t)}{\partial x}\right\|_{1},\left\|\frac{\partial v(x, t)}{\partial x}\right\|_{1}\right\} & \\
& \leq \max \left\{\left\|\frac{\partial u_{0}(x)}{\partial x}\right\|_{1},\left\|\frac{\partial v_{0}(x)}{\partial x}\right\|_{1}\right\} .
\end{aligned}
$$

Let us now consider the case $-1 \leq \alpha<0$. By Proposition 2.2, given any $\delta>0$, we have a unique global solution of system (1.5) in $L^{\infty}(\Omega)$. Furthermore, by Lemma 2.2, given initial data $f_{1}=\left(u_{1}, v_{1}\right)$ and $f_{2}=$ $\left(u_{2}, v_{2}\right)$, with $f_{1}, f_{2} \in D_{\delta}^{+}\left(B_{\alpha}\right)$ the solutions at any subsequent time $t>0$ satisfy inequality $(2.5)$.

As is well-known for accretive nonlinear semigroups, this allows to extend the semigroup to all $L^{1}$-data. In addition, if $u_{0}(x), v_{0}(x)$ have bounded variations, the solution $(u(x, t), v(x, t))$ has bounded variation, and inequality (2.6) holds. The previous arguments are summarized by the following

Proposition 2.3. Let $0 \leq u_{0}(x), v_{0}(x) \in L^{1}(\Omega)$. Then, provided $|\alpha| \leq 1$, the initial-boundary value problem (1.5) has a unique global solution $u(x, t), v(x, t) \in C\left([0, T] ; L^{1}(\Omega)\right)$ for all $T \geq 0$. In addition, if $u_{0}(x), v_{0}(x) \in B V(\Omega)$, then $u(x, t), v(x, t) \in L^{\infty}(0, \infty ; B V(\Omega))$ and (2.10) holds. Furthermore, for any $p \geq 1$, if $u_{0}, v_{0} \in L^{p}(\Omega)$, we have

$$
\left(\int_{\mathbb{R}}\left(u\left(x, t_{2}\right)^{p}+v\left(x, t_{2}\right)^{p}\right) d x\right)^{1 / p} \leq\left(\int_{\mathbb{R}}\left(u\left(x, t_{1}\right)^{p}+v\left(x, t_{1}\right)^{p}\right) d x\right)^{1 / p},
$$

for $t_{1}<t_{2}$. If $0 \leq \alpha \leq 1$, these results extend to $\Omega=\mathbb{R}$.

REMARK 2.2. We emphasize that neither $B V$-bounds, nor $L^{p}$-bounds for the system (1.5) depend on $\varepsilon$. This is not the case if we look for $L^{p}$-bounds on the derivatives.

Indeed we have

Proposition 2.4. Let $0 \leq u_{0}(x), v_{0}(x) \in D^{+}\left(B_{\alpha}\right)$, if $0 \leq \alpha \leq 1$, and $0 \leq u_{0}(x), v_{0}(x) \in D_{\delta}^{+}\left(B_{\alpha}\right)$, for some $\delta>0$, if $\alpha<0$. Then, if $0 \leq u_{0}(x), v_{0}(x) \in W^{m, p}, m \geq 0,1 \leq p \leq \infty, u(x, t), v(x, t) \in W^{m, p}$, 
and, for $t \leq T$

$$
\begin{aligned}
\int_{\Omega}\left(\left|\frac{\partial^{m} u(t)}{\partial x^{m}}\right|^{p}+\left|\frac{\partial^{m} v(t)}{\partial x^{m}}\right|^{p}\right) d x & \\
& \leq c_{m, p}\left(\varepsilon, T, \delta,\|u\|_{\infty},\|v\|_{\infty}\right) \int_{\Omega}\left(\left|\frac{\partial^{m} u_{0}}{\partial x^{m}}\right|^{p}+\left|\frac{\partial^{m} v_{0}}{\partial x^{m}}\right|^{p}\right) d x
\end{aligned}
$$

The case $\alpha=0$ seems to be exceptional. Let us consider the system (1.1) with a constant rate $k=k_{0}$, that is let us consider the GoldsteinTaylor model

$$
\left\{\begin{array}{l}
\frac{\partial u}{\partial t}+c \frac{\partial u}{\partial x}=k_{0}(v-u), \\
\frac{\partial v}{\partial t}-c \frac{\partial v}{\partial x}=k_{0}(u-v), \quad x \in \mathbb{R}, t \geq 0
\end{array}\right.
$$

Easy computations show that

$$
\frac{d}{d t} \int_{\mathbb{R}}\left(\left|\frac{\partial u}{\partial x}\right|^{p}+\left|\frac{\partial v}{\partial x}\right|^{p}\right) d x \leq 0
$$

Hence, combining (2.8) with the result of Proposition 2.3 we conclude that, if the initial data $u_{0}, v_{0} \in W^{1, p}(\mathbb{R}), 1 \leq p \leq \infty$, the solution $u(x, t), v(x, t) \in W^{1, p}(\mathbb{R})$, and

$$
\left(\int_{\mathbb{R}}\left(u^{p}+v^{p}\right) d x\right)^{1 / p}+\left(\int_{\mathbb{R}}\left(\left|\frac{\partial u}{\partial x}\right|^{p}+\left|\frac{\partial v}{\partial x}\right|^{p}\right) d x\right)^{1 / p}
$$

is monotone non increasing in time.

Since the problem is linear, the same conclusion can be reached for higher order derivatives. So, we proved

Proposition 2.5. Let $0 \leq u_{0}(x), v_{0}(x) \in W^{m, p}$, for $m \geq 0,1 \leq p \leq$ $\infty$. Then the unique solution $u(x, t), v(x, t)$ to the initial value problem for the Goldstein-Taylor model (2.14) belongs to $W^{m, p}$ for all $t \geq 0$, and

$$
\left.\sum_{k=0}^{m}\left(\int_{\mathbb{R}}\left|\frac{\partial^{m} u}{\partial x^{m}}\right|^{p}+\left|\frac{\partial v^{m}}{\partial x^{m}}\right|^{p}\right) d x\right)^{1 / p}
$$

is monotone non increasing with time.

To end this section, let us recall that $B_{0}$ is in fact accretive in $L^{p}$ for all $p \in[1, \infty]$. 


\section{Entropy bounds.}

Having in mind the passage to the fluid dynamic limit, we discuss in this section further a priori bounds for the system (1.5), when $\alpha \leq 1$. Let us introduce nonnegative functions $u_{0}(x), v_{0}(x) \in L^{1}(\mathbb{R}) \cap L^{\infty}(\mathbb{R})$ such that

$$
\int_{\mathbb{R}}\left(u_{0}(x)\left|\log u_{0}(x)\right|+v_{0}(x)\left|\log v_{0}(x)\right|\right) d x=M_{1}<\infty
$$

and, for $\omega(x)=\left(1+x^{2}\right)^{\beta / 2}, 0<\beta<1 / 2$,

$$
\int_{\mathbb{R}} \omega(x)\left(u_{0}(x)+v_{0}(x)\right) d x=M_{2}<\infty .
$$

The bounds (3.1) and (3.2) ensure a suitable decay at infinity of $u_{0}$ and $v_{0}$. In section 2 we showed that different values of $\alpha$ produce different results of existence, uniqueness and regularity of the solution to system (1.5). Nevertheless, since we wish to give a unified treatment of our system for any value of $\alpha$, we first maintain our analysis as general as possible, postponing to the end of the section the remarks concerning the various possible extensions of the results for particular values of the parameter $\alpha$.

To this end, given $\gamma>0$, let us denote by $\Omega_{\varepsilon}$, the domain $\left[-1 / \varepsilon^{\gamma}\right.$, $\left.1 / \varepsilon^{\gamma}\right]$. In addition, given $\mu>0$, let

$$
u_{0}^{\varepsilon}=\max \left\{u_{0}(x), \varepsilon^{\mu}\right\}, \quad v_{0}^{\varepsilon}=\max \left\{v_{0}(x), \varepsilon^{\mu}\right\} .
$$

By the results of Section 2, the initial boundary value problem for system (1.5), with periodic boundary conditions on $\Omega_{\varepsilon}$, and initial values (3.3), has a unique global solution $u^{\varepsilon}(x, t), v^{\varepsilon}(x, t)$, for all $\alpha \leq 1$.

Moreover, provided $\mu>\gamma(1+\beta), u_{0}^{\varepsilon}$ and $v_{0}^{\varepsilon}$ satisfy bounds (3.1) and (3.2) with different but finite constants $M_{1}^{\varepsilon}$ and $M_{1}^{\varepsilon}$. In fact we have

$$
\begin{aligned}
& \mid \int_{\Omega_{\varepsilon}}\left(u_{0}^{\varepsilon}(x)\left|\log u_{0}^{\varepsilon}(x)\right|+v_{0}^{\varepsilon}(x)\left|\log v_{0}^{\varepsilon}(x)\right|\right) d x \\
& \quad-\int_{\Omega_{\varepsilon}}\left(u_{0}(x)\left|\log u_{0}(x)\right|+v_{0}(x)\left|\log v_{0}(x)\right|\right) d x \mid \\
& \quad=\mid \int_{\Omega_{\varepsilon} \cap\left\{u_{0}<\varepsilon^{\mu}\right\}}\left(\varepsilon^{\mu}\left|\log \varepsilon^{\mu}\right|-u_{0}(x)\left|\log u_{0}(x)\right|\right) d x
\end{aligned}
$$




$$
\begin{aligned}
& +\int_{\Omega_{\varepsilon} \cap\left\{v_{0}<\varepsilon^{\mu}\right\}}\left(\varepsilon^{\mu}\left|\log \varepsilon^{\mu}\right|-v_{0}(x)\left|\log v_{0}(x)\right|\right) d x \mid \\
\leq & 4 \varepsilon^{\mu}\left|\log \varepsilon^{\mu}\right| \varepsilon^{-\gamma} \\
= & 4 \mu \varepsilon^{\mu-\gamma}|\log \varepsilon|
\end{aligned}
$$

and

$\left|\int_{\Omega_{\varepsilon}} \omega(x)\left(u_{0}^{\varepsilon}(x)+v_{0}^{\varepsilon}(x)\right) d x-\int_{\Omega_{\varepsilon}} \omega(x)\left(u_{0}(x)+v_{0}(x)\right) d x\right|$

$$
\begin{aligned}
& \leq 2 \varepsilon^{\mu} \omega\left(\varepsilon^{\gamma}\right) 2 \varepsilon^{-\gamma} \\
& =4\left(1+\varepsilon^{-2 \gamma}\right)^{\beta / 2} \varepsilon^{\mu-\gamma} .
\end{aligned}
$$

Let us choose $\varphi(r)=r \log r$, for $r \geq 0$. Then, by (2.1) we obtain

$\frac{d}{d t} \int_{\Omega_{\varepsilon}}\left(u^{\varepsilon}(x, t) \log u^{\varepsilon}(x, t)+v^{\varepsilon}(x, t) \log v^{\varepsilon}(x, t)\right) d x$

$$
=-\int_{\Omega_{\varepsilon}}\left(u^{\varepsilon}(x, t)+v^{\varepsilon}(x, t)\right)^{\alpha} \frac{u^{\varepsilon}(x, t)-v^{\varepsilon}(x, t)}{\varepsilon^{2}} \log \frac{u^{\varepsilon}(x, t)}{v^{\varepsilon}(x, t)} d x .
$$

On the other hand, if we multiply both equations (1.5) by $\omega$, after integrating over $\Omega_{\varepsilon}$, we get

$$
\begin{aligned}
\frac{d}{d t} \int_{\Omega_{\varepsilon}} \omega(x)\left(u^{\varepsilon}(x, t)+\right. & \left.v^{\varepsilon}(x, t)\right) d x \\
& +\frac{1}{\varepsilon} \int_{\Omega_{\varepsilon}} \omega(x) \frac{\partial}{\partial x}\left(u^{\varepsilon}(x, t)-v^{\varepsilon}(x, t)\right) d x=0 .
\end{aligned}
$$

Integrating by parts, and making use of the periodicity, we deduce

$$
\begin{aligned}
\frac{d}{d t} \int_{\Omega_{\varepsilon}} \omega(x)\left(u^{\varepsilon}(x, t)+\right. & \left.v^{\varepsilon}(x, t)\right) d x \\
& -\frac{1}{\varepsilon} \int_{\Omega_{\varepsilon}} \omega^{\prime}(x) \frac{u^{\varepsilon}(x, t)-v^{\varepsilon}(x, t)}{\varepsilon} d x=0
\end{aligned}
$$


Then, taking the sum of (3.6) and (3.7) we conclude

$$
\begin{gathered}
\frac{d}{d t} \int_{\Omega_{\varepsilon}}\left(u^{\varepsilon}(x, t) \log u^{\varepsilon}(x, t)+v^{\varepsilon}(x, t) \log v^{\varepsilon}(x, t)\right. \\
\left.+\omega(x)\left(u^{\varepsilon}(x, t)+v^{\varepsilon}(x, t)\right)\right) d x \\
+\int_{\Omega_{\varepsilon}}\left(u^{\varepsilon}(x, t)+v^{\varepsilon}(x, t)\right)^{\alpha} \frac{u^{\varepsilon}(x, t)-v^{\varepsilon}(x, t)}{\varepsilon^{2}} \log \frac{u^{\varepsilon}(x, t)}{v^{\varepsilon}(x, t)} d x \\
\leq \int_{\Omega_{\varepsilon}}\left|\omega^{\prime}(x) \frac{u^{\varepsilon}(x, t)-v^{\varepsilon}(x, t)}{\varepsilon}\right| d x .
\end{gathered}
$$

Let $\rho_{\varepsilon}=u^{\varepsilon}+v^{\varepsilon}$, and by $j_{\varepsilon}=\left(u^{\varepsilon}(x, t)-v^{\varepsilon}(x, t)\right) / \varepsilon$. Then, for $0<\theta<1$, we obtain

$$
\begin{aligned}
\rho_{\varepsilon}^{\alpha} \frac{u^{\varepsilon}-v^{\varepsilon}}{\varepsilon^{2}} \log \frac{u^{\varepsilon}}{v^{\varepsilon}} & =\rho_{\varepsilon}^{\alpha} j_{\varepsilon}^{2} \frac{\log u^{\varepsilon}-\log v^{\varepsilon}}{u^{\varepsilon}-v^{\varepsilon}} \\
& =\rho_{\varepsilon}^{\alpha} j_{\varepsilon}^{2} \frac{1}{\theta u^{\varepsilon}+(1-\theta) v^{\varepsilon}} \\
& =\rho_{\varepsilon}^{\alpha-1} j_{\varepsilon}^{2} \frac{u^{\varepsilon}+v^{\varepsilon}}{\theta u^{\varepsilon}+(1-\theta) v^{\varepsilon}} \\
& \geq 2 \rho_{\varepsilon}^{\alpha-1} j_{\varepsilon}^{2} .
\end{aligned}
$$

Now, by the a priori bound (2.3),

$$
\rho_{\varepsilon} \leq 2 \max \left\{\left\|u_{0}\right\|_{\infty},\left\|v_{0}\right\|_{\infty}\right\}=\nu\left(u_{0}, v_{0}\right) .
$$

Hence, since $\alpha-1 \leq 0$,

$$
\rho_{\varepsilon}^{\alpha} \frac{u^{\varepsilon}-v^{\varepsilon}}{\varepsilon^{2}} \log \frac{u^{\varepsilon}}{v^{\varepsilon}} \geq 2 \nu^{\alpha-1} j_{\varepsilon}^{2} .
$$

In all cases we obtained a bound from below in terms of $\nu\left(u_{0}, v_{0}\right)$, which depends only on the $L^{\infty}$-norm of the initial values, and not on $\varepsilon$.

Let us now consider the case $-1 \leq \alpha<0$. Then, by (3.9) it follows

$$
\rho_{\varepsilon}^{\alpha} \frac{u^{\varepsilon}-v^{\varepsilon}}{\varepsilon^{2}} \log \frac{u^{\varepsilon}}{v^{\varepsilon}} \geq 2 \rho_{\varepsilon}^{\alpha-1} j_{\varepsilon}^{2} \geq 2 \rho_{\varepsilon}^{2 \alpha} j_{\varepsilon}^{2} \nu^{|\alpha|-1} .
$$

We next observe that

$$
\begin{aligned}
\int_{\Omega_{\varepsilon}} \omega^{\prime}\left|j_{\varepsilon}\right| d x & =\frac{1}{2} \int_{\Omega_{\varepsilon}} \frac{\omega^{\prime}}{\sqrt{\nu}}\left|2 \sqrt{\nu} j_{\varepsilon}\right| d x \\
& \leq \frac{\nu}{2} \int_{\Omega_{\varepsilon}} j_{\varepsilon}^{2} d x+\frac{1}{2 \nu} \int_{\Omega_{\varepsilon}}\left(\omega^{\prime}\right)^{2} d x .
\end{aligned}
$$


By definition, since $0<\beta<1 / 2, \omega^{\prime} \in L^{2}(\mathbb{R})$. Thus, by (3.8) we deduce

$$
\begin{aligned}
& \frac{d}{d t} \int_{\Omega_{\varepsilon}}\left(u^{\varepsilon}(x, t) \log u^{\varepsilon}(x, t)\right.+v^{\varepsilon}(x, t) \log v^{\varepsilon}(x, t) \\
&\left.+\omega(x)\left(u^{\varepsilon}(x, t)+v^{\varepsilon}(x, t)\right)\right) d x+\frac{\nu}{2} \int_{\Omega_{\varepsilon}} j_{\varepsilon}^{2}(x, t) d x \\
& \leq \frac{1}{2 \nu} \int_{\Omega_{\varepsilon}}\left(\omega^{\prime}\right)^{2}(x) d x
\end{aligned}
$$

In particular, for any $t \geq 0$

$$
\begin{aligned}
\int_{\Omega_{\varepsilon}}\left(u^{\varepsilon}(t)\right. & \left.\log u^{\varepsilon}(t)+v^{\varepsilon}(t) \log v^{\varepsilon}(t)+\omega\left(u^{\varepsilon}(t)+v^{\varepsilon}(t)\right)\right) d x \\
\leq & \frac{t}{2 \nu} \int_{\Omega_{\varepsilon}}\left(\omega^{\prime}\right)^{2}(x) d x+\int_{\Omega_{\varepsilon}} \omega\left(u_{0}^{\varepsilon}(t)+v_{0}^{\varepsilon}(t)\right) d x \\
& +\int_{\Omega_{\varepsilon}}\left(u_{0}^{\varepsilon}(t) \log u_{0}^{\varepsilon}(t)+v_{0}^{\varepsilon}(t) \log v_{0}^{\varepsilon}(t)\right) d x \\
\leq & \frac{t}{2 \nu} \int_{\mathbb{R}}\left(\omega^{\prime}\right)^{2}(x) d x+\int_{\mathbb{R}} \omega\left(u_{0}+v_{0}\right) d x \\
& +\int_{\Omega_{\varepsilon}}\left(u_{0} \log u_{0}+v_{0} \log v_{0}\right) d x+o(\varepsilon)
\end{aligned}
$$

where the rest $o(\varepsilon)$ is given by the sum of the right-hand sides of (3.4) and (3.5). By (3.15), the monotonicity of

$$
\int_{\Omega_{\varepsilon}}\left(u^{\varepsilon}(t) \log u^{\varepsilon}(t)+v^{\varepsilon}(t) \log v^{\varepsilon}(t)\right) d x
$$

implies

$$
\begin{aligned}
\int_{\Omega_{\varepsilon}} \omega\left(u^{\varepsilon}(t)+v^{\varepsilon}(t)\right) d x \leq & \frac{t}{2 \nu} \int_{\mathbb{R}}\left(\omega^{\prime}\right)^{2}(x) d x+\int_{\mathbb{R}} \omega\left(u_{0}+v_{0}\right) d x \\
& +\int_{\mathbb{R}}\left(u_{0}(x)\left|\log u_{0}(x)\right|+v_{0}(x)\left|\log v_{0}(x)\right|\right) d x \\
& +\int_{\Omega_{\varepsilon}}\left(u^{\varepsilon}(t) \log ^{-} u^{\varepsilon}(t)+v^{\varepsilon}(t) \log ^{-} v^{\varepsilon}(t)\right) d x \\
& +o(\varepsilon)
\end{aligned}
$$


where $\log ^{-} r$ denotes the negative part of the logarithm.

By the classical inequality $z \log ^{-} z \leq y-z \log y, 0<z, y \leq 1$, choosing $y=\exp (-\omega(x) / 2)$, and $z=u_{\varepsilon}(x)$, we obtain

$$
\begin{aligned}
& \int_{\Omega_{\varepsilon}} u^{\varepsilon} \log ^{-} u^{\varepsilon} d x \leq \int_{\mathbb{R}} \exp \left(-\frac{\omega(x)}{2}\right) d x+\frac{1}{2} \int_{\Omega_{\varepsilon}} \omega u^{\varepsilon} d x, \\
& \int_{\Omega_{\varepsilon}} v^{\varepsilon} \log ^{-} v^{\varepsilon} d x \leq \int_{\mathbb{R}} \exp \left(-\frac{\omega(x)}{2}\right) d x+\frac{1}{2} \int_{\Omega_{\varepsilon}} \omega v^{\varepsilon} d x .
\end{aligned}
$$

Finally, for any $\varepsilon \leq 1$, making use of inequalities (3.17) on the righthand side of (3.16) we obtain

$\frac{1}{2} \int_{\Omega_{\varepsilon}} \omega\left(u^{\varepsilon}(t)+v^{\varepsilon}(t)\right) d x$

$$
\begin{aligned}
\leq & \frac{t}{2 \nu} \int_{\mathbb{R}}\left(\omega^{\prime}\right)^{2}(x) d x+2 \int_{\mathbb{R}} \exp \left(-\frac{\omega(x)}{2}\right) d x+\int_{\mathbb{R}} \omega\left(u_{0}+v_{0}\right) d x \\
& +\int_{\mathbb{R}}\left(u_{0}(x)\left|\log u_{0}(x)\right|+v_{0}(x)\left|\log v_{0}(x)\right|\right) d x+c .
\end{aligned}
$$

In conclusion, for any $t \geq 0$ we obtained the bound

$$
\int_{\Omega_{\varepsilon}} \omega\left(u^{\varepsilon}(t)+v^{\varepsilon}(t)\right) d x \leq c_{1}\left(t, u_{0}, v_{0}\right)
$$

where the constant $c_{1}$ does not depend on $\varepsilon$. By applying (3.19) into (3.17) we obtain an upper bound for the negative part of $u^{\varepsilon}(t) \log ^{-} u^{\varepsilon}(t)$ $+v^{\varepsilon}(t) \log ^{-} v^{\varepsilon}(t)$ in terms of $c_{1}$. Hence, if the initial data satisfy conditions (3.1) and (3.2), for any $T>0$ there exists a constant $C_{T}$, depending only on $u_{0}$ and $v_{0}$, such that, for all $t \leq T$ and $\varepsilon>0$,

$$
\begin{aligned}
& \int_{\Omega_{\varepsilon}}\left(u^{\varepsilon}(t)\left|\log u^{\varepsilon}(t)\right|\right. \\
& \left.\quad+v^{\varepsilon}(t)\left|\log v^{\varepsilon}(t)\right|+\omega\left(u^{\varepsilon}(t)+v^{\varepsilon}(t)\right)\right) d x \leq C_{T} .
\end{aligned}
$$

Now, by (3.14) we argue that for any $\alpha \leq 1$ and $T>0, j_{\varepsilon}$ is bounded in $L^{2}\left([0, T] \times \Omega_{\varepsilon}\right)$. In addition, in view of $(3.9)$, if $\alpha \leq 1, \rho_{\varepsilon}^{(\alpha-1) / 2} j_{\varepsilon}$ is bounded in $L^{2}\left([0, T] \times \Omega_{\varepsilon}\right)$. Next, if $|\alpha| \leq 1, \rho_{\varepsilon}^{\alpha} j_{\varepsilon}$ is bounded in $L^{2}\left([0, T] \times \Omega_{\varepsilon}\right)$. This follows by (3.12) when $\alpha<0$, and by (3.11) and the $L^{\infty}$-bound (3.10) when $\alpha \geq 0$. 
By the Propositions 2.3 and 2.4, it follows that the previous bounds can be extended to all initial data satisfying (3.1) and (3.2) and to all of $\mathbb{R}$ when $\alpha$ is positive, to all initial data satisfying (3.1) and (3.2) and to any domain $\Omega_{\varepsilon}$ when $-1 \leq \alpha \leq 0$. In conclusion we proved

Theorem 3.1. Let $0 \leq u_{0}, v_{0} \in L^{1}(\mathbb{R}) \cap L^{\infty}(\mathbb{R})$ satisfy conditions (3.1) and (3.2).

i) Let $0 \leq \alpha \leq 1$. Then, for all $T>0$, and $\varepsilon>0$ there exist constants $d_{1}=d_{1}\left(u_{0}, v_{0}, T\right), d_{2}=d_{2}\left(u_{0}, v_{0}, T\right)$ and $d_{3}=d_{3}\left(u_{0}, v_{0}, T\right)$ such that, the unique solution $u^{\varepsilon}(x, t), v^{\varepsilon}(x, t)$ of the initial value problem for system (1.5) satisfies

$$
\begin{gathered}
\int_{0}^{T} \int_{\mathbb{R}}\left(\frac{u^{\varepsilon}(x, t)-v^{\varepsilon}(x, t)}{\varepsilon}\right)^{2} d x d t \leq d_{1}, \\
\int_{0}^{T} \int_{\mathbb{R}}\left(\frac{u^{\varepsilon}(x, t)-v^{\varepsilon}(x, t)}{\varepsilon}\right)^{2} \\
\cdot\left(u^{\varepsilon}(x, t)+v^{\varepsilon}(x, t)\right)^{\alpha-1} d x d t \leq d_{2}, \\
\int_{\mathbb{R}}\left(u^{\varepsilon}(t)\left|\log u^{\varepsilon}(t)\right|\right. \\
\left.+v^{\varepsilon}(t)\left|\log v^{\varepsilon}(t)\right|+\omega\left(u^{\varepsilon}(t)+v^{\varepsilon}(t)\right)\right) d x \leq d_{3} .
\end{gathered}
$$

ii) Let $-1 \leq \alpha<0$. Then, for all $T>0$, and $k \in \mathbb{N}^{+}$, the unique solution $u^{\varepsilon}(x, t), v^{\varepsilon}(x, t)$ of the initial-boundary value problem for system (1.5) on the domain $\left[-1 / \varepsilon^{k}, 1 / \varepsilon^{k}\right]$ satisfies

$$
\begin{gathered}
\int_{0}^{T} \int_{-1 / \varepsilon^{k}}^{1 / \varepsilon^{k}}\left(\frac{u^{\varepsilon}(x, t)-v^{\varepsilon}(x, t)}{\varepsilon}\right)^{2} d x d t \leq d_{1}, \\
\int_{0}^{T} \int_{-1 / \varepsilon^{k}}^{1 / \varepsilon^{k}}\left(\frac{u^{\varepsilon}(x, t)-v^{\varepsilon}(x, t)}{\varepsilon}\right)^{2} \\
\cdot\left(u^{\varepsilon}(x, t)+v^{\varepsilon}(x, t)\right)^{2 \alpha} d x d t \leq d_{2}, \\
\int_{-1 / \varepsilon^{k}}^{1 / \varepsilon^{k}}\left(u^{\varepsilon}(t)\left|\log u^{\varepsilon}(t)\right|\right. \\
\left.+v^{\varepsilon}(t)\left|\log v^{\varepsilon}(t)\right|+\omega\left(u^{\varepsilon}(t)+v^{\varepsilon}(t)\right)\right) d x \leq d_{3} .
\end{gathered}
$$


iii) Let $\alpha<-1$. Given $k \in \mathbb{N}^{+}$, let $\mu>k(1+\beta)$, and let $u_{0}^{\varepsilon}$, $v_{0}^{\varepsilon}$ be defined by (3.3). Then, for all $T>0$, the unique solution of the initialboundary value problem for system (1.5) on the domain $\left[-1 / \varepsilon^{k}, 1 / \varepsilon^{k}\right]$, with initial data $u_{0}^{\varepsilon}, v_{0}^{\varepsilon}$, satisfies the bounds (3.24), (3.26) and

$$
\begin{aligned}
\int_{0}^{T} \int_{-1 / \varepsilon^{k}}^{1 / \varepsilon^{k}} & \left(\frac{u^{\varepsilon}(x, t)-v^{\varepsilon}(x, t)}{\varepsilon}\right)^{2} \\
& \cdot\left(u^{\varepsilon}(x, t)+v^{\varepsilon}(x, t)\right)^{2 \tau} d x d t \leq d_{2}
\end{aligned}
$$

for all $0<\tau \leq(|\alpha|+1) / 2$.

REMARK 3.1. Let $\alpha<0$. Given $\varepsilon>0$, the velocity of propagation of the hyperbolic system (1.5) is exactly $1 / \varepsilon$. This means that, given any time $T>0$, for $t \leq T$ the solution $u^{\varepsilon}(x, t), v^{\varepsilon}(x, t)$ on the interval $[-(1+T) / \varepsilon,(1+T) / \varepsilon]$ depends only on the initial values on the interval $[-(1+2 T) / \varepsilon,(1+2 T) / \varepsilon]$, provided the boundaries are located at $\left[-1 / \varepsilon^{k}, 1 / \varepsilon^{k}\right]$, for $k$ large enough. In other words, for $\varepsilon$ small enough, the presence of the boundaries does not affect the solution on the interval $[-(1+T) / \varepsilon,(1+T) / \varepsilon]$. This explain why the presence of the boundaries does not affect the solution in any bounded set in the limit procedure.

\section{Limit theorems.}

The macroscopic equations for the system (1.5) can be expressed in terms of the mass density $\rho_{\varepsilon}(x, t)$ and of the rescaled flux

$$
j_{\varepsilon}(x, t)=\frac{u_{\varepsilon}(x, t)-v_{\varepsilon}(x, t)}{\varepsilon}
$$

as follows

$$
\left\{\begin{array}{l}
\frac{\partial \rho_{\varepsilon}}{\partial t}+\frac{\partial j_{\varepsilon}}{\partial x}=0 \\
\varepsilon^{2} \frac{\partial j_{\varepsilon}}{\partial t}+\frac{\partial \rho_{\varepsilon}}{\partial x}=-2 \rho_{\varepsilon}^{\alpha} j_{\varepsilon}, \quad x \in \mathbb{R}, t \geq 0 .
\end{array}\right.
$$

In this section, we study the limiting behaviour, as $\varepsilon$ goes to zero, of the solutions $\left(\rho_{\varepsilon}, j_{\varepsilon}\right)$ to system (4.2). In our passage to the limit, we will consider various relatively compact sequences. In these cases, without 
risk of misunderstanding, when we say that the sequence converges to a limit, we mean that there exists a subsequence that converges to a limit.

In the rest of the section, we will consider initial values for the kinetic problem (1.5) satisfying the conditions of Theorem 3.1.

Let us first consider the case $0 \leq \alpha<1$. In this case, by Proposition 2.3 , if the initial values $u_{0}, v_{0}$ belong to $L^{1} \cap L^{\infty},(2.9)$ holds. Hence we see that, for all $T>0$

$$
\lim _{h \rightarrow 0} \sup _{0 \leq t \leq T}\left\|\rho_{\varepsilon}(x+h, t)-\rho_{\varepsilon}(x, t)\right\|_{1}=0 .
$$

Moreover, by (3.23) we deduce that

$$
\lim _{R \rightarrow \infty} \sup _{t \in[0, T]} \int_{|x|>R} \rho_{\varepsilon}(x, t) d x=0 .
$$

In addition, by Theorem 3.1, $\left\{j_{\varepsilon}\right\}$ is bounded in $L^{2}$, and thus by (4.2) $\partial \rho_{\varepsilon} / \partial t$ is bounded in $L^{2}\left(0, T ; H_{\mathrm{loc}}^{-1}\right)$ for all $T>0$. In view of the bound (2.3) $\left\{\rho_{\varepsilon}\right\}$ is bounded in $L^{\infty}(\mathbb{R})$.

These bounds, combined with (4.3) and (4.4), imply that the family $\left\{\rho_{\varepsilon}\right\}$ is relatively compact in $C\left([0, T] ; L^{1}(\mathbb{R})\right)$ for all $T>0$.

Hence $\rho_{\varepsilon}^{\alpha} j_{\varepsilon} \longrightarrow \rho^{\alpha} j$ in $L^{2}$-weak, $\varepsilon^{2} j_{\varepsilon} \longrightarrow 0$ strongly in $L^{2}$ and from the flux equation (4.2) we deduce

$$
\frac{\partial \rho}{\partial x}=-2 \rho^{\alpha} j,
$$

at least in the sense of distributions (and in fact in $L^{2}$ ). Considering that $\rho \in L^{\infty},(4.5)$ implies that we have, at least formally

$$
j=-\frac{1}{2(1-\alpha)} \frac{\partial \rho^{1-\alpha}}{\partial x}
$$

in $L^{2}$. This fact follows indeed from (4.5), since $\rho \in H_{x}^{1}$ and thus

$$
\frac{\partial}{\partial x} \frac{(\varepsilon+\rho)^{1-\alpha}}{1-\alpha}=-2 \frac{\rho^{\alpha}}{(\varepsilon+\rho)^{\alpha}} j
$$

which converges in $L^{2}$ to $-2 j \mathbf{1}_{\{\rho>0\}}$. Therefore, $\rho^{1-\alpha} \in H_{x}^{-1}$, and (4.6) holds on the set $\{\rho>0\}$. In addition, the entropy bound (3.22) shown in Section 3 implies at the limit that $\rho^{(\alpha-1) / 2} j \in L^{2}(\mathbb{R} \times(0, T))$ 
for all $T>0$. Thus, if $\alpha<1, j=0$, almost everywhere on $\rho=0$, almost everywhere, and the proof of (4.6) is complete.

If we now replace (4.6) in the continuity equation, we recover that the limit density $\rho$ satisfies the fast diffusion equation

$$
\frac{\partial \rho}{\partial t}-\frac{1}{2(1-\alpha)} \frac{\partial^{2} \rho^{1-\alpha}}{\partial x^{2}}=0 .
$$

Since we assumed the initial values $u_{0}, v_{0} \in L^{1} \cap L^{\infty}$, so is the initial density $\rho_{0}(x)=\rho(x, t=0)$. On the other hand, the fast diffusion equation (4.7) has a unique global solution in $D^{\prime}$, provided $\rho(0, x) \in$ $L_{\text {loc }}^{1}(\mathbb{R})$ (cf. M.A. Herrero and M. Pierre $[\mathrm{HePi}]$ ). The uniqueness result guarantees the existence of a unique limit point for the whole family.

Hence, we obtained the result of [PTo] as a particular case $(\alpha>0)$ of the limit behaviour of the system (4.2).

Theorem 4.1. Let $0 \leq \alpha<1$, and let $\left(\rho_{\varepsilon}, j_{\varepsilon}\right)$ be a sequence of solutions to the initial value problem for the system (4.2), where the initial values $u_{0}, v_{0}$ satisfy the hypotheses of Proposition 2.3 and Theorem 3.1. Then, there exists $\rho \in L^{1} \cap L^{\infty}$ such that $\rho_{\varepsilon}(x, t)$ converges to $\rho(x, t)$ strongly in $C\left([0, T] ; L^{1}(\mathbb{R})\right)$ for all $T \geq 0$, while $\varepsilon j_{\varepsilon}$ converges to zero strongly in $L^{2}(\mathbb{R} \times[0, T])$. The limit density $\rho(x, t)$ is the (unique) weak solution to the Cauchy problem for the fast diffusion equation $(4.7)$, in $D^{\prime}(\mathbb{R}) \times$ $(0, \infty)$, with initial datum $\rho_{0}=u_{0}+v_{0}$.

The proof for the case $-1 \leq \alpha<0$ is similar. By Theorem 3.1, we deduce that $n_{\varepsilon}=\rho_{\varepsilon}^{\alpha} j_{\varepsilon}$ converges to $v$ in $L^{2}$-weak, while $\rho_{\varepsilon}$ is relatively compact in $C\left([0, T] ; L_{\text {loc }}^{1}(\mathbb{R})\right)$ for all $T>0$. In addition $\varepsilon^{2} j_{\varepsilon} \rightarrow 0$ strongly in $L^{2}$, exactly as in the case $\alpha>0$. Let us rewrite the second equation of system (4.2) in the form

$$
\varepsilon^{2} \frac{\partial j_{\varepsilon}}{\partial t}+\frac{\partial \rho_{\varepsilon}}{\partial x}=-2 n_{\varepsilon} .
$$

Passing to the limit in (4.8), we deduce

$$
n=-\frac{1}{2} \frac{\partial \rho}{\partial x}
$$

in $D^{\prime}$. Now, from the continuity equation, considering that

$$
j_{\varepsilon}=\rho_{\varepsilon}^{|\alpha|} n_{\varepsilon} \longrightarrow-\frac{\rho^{|\alpha|}}{2} \frac{\partial \rho}{\partial x}
$$


in $L^{2}$-weak, we obtain that the limit density satisfies (in a weak sense) the slow diffusion equation

$$
\frac{\partial \rho}{\partial t}-\frac{1}{2(1+|\alpha|)} \frac{\partial^{2} \rho^{1+|\alpha|}}{\partial x^{2}}=0 .
$$

We proved

Theorem 4.2. Let $-1 \leq \alpha<0$, and let $\left(\rho_{\varepsilon}, j_{\varepsilon}\right)$ be a sequence of solutions to the initial-boundary value problem for the system (4.2), where the initial values $u_{0}, v_{0}$ satisfy the hypotheses of Proposition 2.3 and Theorem 3.1. Then, there exists $\rho \in L_{\mathrm{loc}}^{1} \cap L^{\infty}$ such that $\rho_{\varepsilon}(x, t)$ converges to $\rho(x, t)$ in $C\left([0, T] ; L_{\mathrm{loc}}^{1}(\mathbb{R})\right)$ for all $T \geq 0$, while $\varepsilon j_{\varepsilon}(x, t)$ converges to zero strongly in $L_{\mathrm{loc}}^{2}(\mathbb{R} \times[0, T])$. The limit density $\rho(x, t)$ is the (unique) weak solution to the Cauchy problem for the slow diffusion equation $(4.10)$, in $D^{\prime}((0, \infty) \times \mathbb{R})$, with initial datum $\rho_{0}=u_{0}+v_{0}$.

REMARK 4.1. An easy consequence of the previous results is that both $u_{\varepsilon}(x, t)$ and $v_{\varepsilon}(x, t)$, solutions to the initial (if $0 \leq \alpha<1$ ) (or initialboundary (if $-1 \leq \alpha<0$ )) value problem for the kinetic system (1.5) converge strongly to $\rho / 2$, where $\rho$ is the solution of the corresponding nonlinear diffusion equation.

We will now examine the case $\alpha<-1$. The main argument in our proof of the passage to the limit will be the "div-curl" lemma of compensated compactness theory (see F. Murat [Mu1], [Mu2] and L. Tartar [Ta1], [Ta2]).

Lemma 4.1. Let $A$ be an open set of $\mathbb{R}^{n}$, and $v_{\varepsilon}$ and $w_{\varepsilon}$ be two sequences such that

$$
\begin{aligned}
& v_{\varepsilon} \longrightarrow v, \quad \text { in }\left[L^{2}(A)\right]^{n} \text {-weak }, \\
& w_{\varepsilon} \longrightarrow w, \quad \text { in }\left[L^{2}(A)\right]^{n}-\text { weak },
\end{aligned}
$$

$$
\operatorname{curl} w_{\varepsilon} \text { is bounded in }\left[L^{2}(A)\right]^{n} \text { (or compact in }\left[H^{-1}(A)\right]^{n^{2}} \text { ). }
$$

Let $\langle\cdot, \cdot\rangle$ denote the inner product in $\mathbb{R}^{n}$, i.e. $\langle v, w\rangle=\sum_{i=1}^{n} v_{i} w_{i}$. Then

$$
\left\langle v_{\varepsilon}, w_{\varepsilon}\right\rangle \longrightarrow\langle v, w\rangle \text { in } D^{\prime} .
$$


Let us take initial data satisfying part iii) of Theorem 3.1. Since, with these hypotheses, Proposition 2.2 holds, $\rho_{\varepsilon} \geq 2 \varepsilon^{\mu}$, and, for given $n \in \mathbb{N}$, $n \geq 1$, the system (4.2) is equivalent to

$$
\left\{\begin{array}{l}
\frac{\partial \rho_{\varepsilon}}{\partial t}+\frac{\partial j_{\varepsilon}}{\partial x}=0 \\
\varepsilon^{2} \rho_{\varepsilon}^{n} \frac{\partial j_{\varepsilon}}{\partial t}+\frac{1}{n+1} \frac{\partial \rho_{\varepsilon}^{n+1}}{\partial x}=-2 \rho_{\varepsilon}^{\alpha+n} j_{\varepsilon}
\end{array}\right.
$$

The second equation of the system (4.14) can be written as follows

$$
\frac{\partial}{\partial t}\left(\varepsilon^{2} \rho_{\varepsilon}^{n} j_{\varepsilon}\right)+\frac{1}{n+1} \frac{\partial \rho_{\varepsilon}^{n+1}}{\partial x}-n \varepsilon^{2} j_{\varepsilon} \rho_{\varepsilon}^{n-1} \frac{\partial \rho_{\varepsilon}}{\partial t}=-2 \rho_{\varepsilon}^{\alpha+n} j_{\varepsilon} .
$$

Making use of the continuity equation, we deduce

$$
\begin{aligned}
-n \varepsilon^{2} j_{\varepsilon} \rho_{\varepsilon}^{n-1} \frac{\partial \rho_{\varepsilon}}{\partial t} & =\frac{n}{2} \varepsilon^{2} \rho_{\varepsilon}^{n-1} \frac{\partial j_{\varepsilon}^{2}}{\partial x} \\
& =\frac{n}{2} \varepsilon^{2} \frac{\partial}{\partial x}\left(\rho_{\varepsilon}^{n-1} j_{\varepsilon}^{2}\right)-\frac{n(n-1)}{2} \varepsilon^{2} \rho_{\varepsilon}^{n-2} j_{\varepsilon}^{2} \frac{\partial \rho_{\varepsilon}}{\partial x} .
\end{aligned}
$$

Hence, choosing $n=1$, we conclude that the system (4.14) is equivalent to

$$
\left\{\begin{array}{l}
\frac{\partial \rho_{\varepsilon}}{\partial t}+\frac{\partial j_{\varepsilon}}{\partial x}=0 \\
\frac{\partial}{\partial t}\left(\varepsilon^{2} \rho_{\varepsilon} j_{\varepsilon}\right)+\frac{\partial}{\partial x}\left(\frac{\rho_{\varepsilon}^{2}}{2}+\frac{\varepsilon^{2}}{2} j_{\varepsilon}^{2}\right)=-2 \rho_{\varepsilon}^{\alpha+1} j_{\varepsilon} .
\end{array}\right.
$$

If $n>1$, we substitute the result of (4.16) into (4.15) to obtain

$$
\begin{aligned}
\frac{\partial}{\partial t}\left(\varepsilon^{2} \rho_{\varepsilon}^{n} j_{\varepsilon}\right)+\frac{\partial}{\partial x}\left(\frac{\rho_{\varepsilon}^{n+1}}{n+1}+\varepsilon^{2} \frac{n}{2} \rho_{\varepsilon}^{n-1} j_{\varepsilon}^{2}\right)-\varepsilon^{2} \frac{n(n-1)}{2} j_{\varepsilon}^{2} \rho_{\varepsilon}^{n-2} \frac{\partial \rho_{\varepsilon}}{\partial x} \\
=-2 \rho_{\varepsilon}^{\alpha+n} j_{\varepsilon} .
\end{aligned}
$$

The term $\partial \rho_{\varepsilon} / \partial x$ in (4.18) can be evaluated by the second equation of the system (4.2), to give

$$
\begin{aligned}
& -\varepsilon^{2} \frac{n(n-1)}{2} j_{\varepsilon}^{2} \rho_{\varepsilon}^{n-2} \frac{\partial \rho_{\varepsilon}}{\partial x} \\
& =n(n-1) \varepsilon^{2} j_{\varepsilon}^{3} \rho_{\varepsilon}^{-2+\alpha}+\varepsilon^{4} \frac{n(n-1)}{6} \rho_{\varepsilon}^{n-2} \frac{\partial j_{\varepsilon}^{3}}{\partial t}
\end{aligned}
$$


If we now take $n=2$, we find that the second equation of system (4.2) can be written as follows

$$
\begin{aligned}
\frac{\partial}{\partial t}\left(\varepsilon^{2} \rho_{\varepsilon}^{2} j_{\varepsilon}+\varepsilon^{4} \frac{j_{\varepsilon}^{3}}{3}\right)+\frac{\partial}{\partial x}( & \left.\frac{\rho_{\varepsilon}^{3}}{3}+\varepsilon^{2} \rho_{\varepsilon} j_{\varepsilon}^{2}\right) \\
& =-2 \rho_{\varepsilon}^{\alpha+2} j_{\varepsilon}-2 \varepsilon^{2} j_{\varepsilon}^{3} \rho_{\varepsilon}^{-2+\alpha} .
\end{aligned}
$$

The general formula follows easily by induction. In particular, consider that, given $\alpha<-2$, there exists $n \in \mathbb{N}$ such that $\tau=|\alpha|-2 n \leq$ $(|\alpha|+1) / 2$. This implies, by Theorem 3.1, that $\rho_{\varepsilon}^{-\tau} j_{\varepsilon}$ is bounded in $L^{2}$. With this choice, the second equation of the system (4.14) can be written in equivalent form as

$$
\begin{aligned}
\frac{\partial}{\partial t}\left(\varepsilon^{2} \sum_{k=0}^{n} a_{n, k} \varepsilon^{2 k} \rho_{\varepsilon}^{2(n-k)} j_{\varepsilon}^{2 k+1}\right) & +\frac{\partial}{\partial x}\left(\frac{\rho_{\varepsilon}^{2 n+1}}{2 n+1}+\sum_{k=1}^{n} b_{n, k} \varepsilon^{2 k} \rho_{\varepsilon}^{2(n-k)+1} j_{\varepsilon}^{2 k}\right) \\
& =-2\left(\rho_{\varepsilon}^{-\tau} j_{\varepsilon}+\sum_{k=1}^{n} c_{n, k} \varepsilon^{2 k} \rho_{\varepsilon}^{-\tau-2 k} j_{\varepsilon}^{2 k+1}\right)
\end{aligned}
$$

where $a_{n, k}, b_{n, k}$ and $c_{n, k}$ are suitable bounded constants that can be computed explicitly.

The previous argument allows us to prove the following

Proposition 4.1. Let $0 \leq u_{0}, v_{0}$ satisfy the hypotheses of Theorem 3.1. Given $\alpha<-1$, let $n \in \mathbb{N}$ be such that $0 \leq \tau=|\alpha|-2 n \leq(|\alpha|+1) / 2$. Then, if $\rho=w^{*}-\lim \rho_{\varepsilon}$ in $L^{\infty}$,

$$
\begin{aligned}
& \rho_{\varepsilon}^{2 n+1} \longrightarrow \rho^{2 n+1}, \quad \text { in } D^{\prime}, \\
& \rho_{\varepsilon} \longrightarrow \rho, \quad \text { in } L_{\mathrm{loc}}^{p} \text { for all } 1 \leq p<\infty .
\end{aligned}
$$

Proof. Let us set $U_{\varepsilon}=\left(\rho_{\varepsilon}, j_{\varepsilon}\right)$. The continuity equation (4.2) becomes $\left\{\operatorname{div} U_{\varepsilon}\right\}=0$. Given any region $A \Subset \mathbb{R} \times \mathbb{R}^{+}$, from equation (4.21) we see that the right-hand side is bounded in $L^{2}(A)$. In fact, by definition of $j_{\varepsilon},\left|j_{\varepsilon} / \rho_{\varepsilon}\right| \leq 1 / \varepsilon$. Thus

$$
\int_{A}\left(\varepsilon^{2 k} \rho_{\varepsilon}^{-\tau-2 k} j_{\varepsilon}^{2 k+1}\right)^{2} d x d t \leq \int_{A} \rho_{\varepsilon}^{-2 \tau} j_{\varepsilon}^{2} d x d t
$$

and the above integral is bounded in view of (3.27). Moreover

$$
\begin{aligned}
\int_{A}\left|\varepsilon^{2 k} \rho_{\varepsilon}^{-\tau-2 k} j_{\varepsilon}^{2 k+1}\right| d x d t & =\varepsilon^{2 k} \int_{A} \rho_{\varepsilon}^{-\tau-1} j_{\varepsilon}^{2}\left|\frac{j_{\varepsilon}}{\rho_{\varepsilon}}\right|^{2 k-1} d x d t \\
& \leq \varepsilon \int_{A} \rho_{\varepsilon}^{-\tau-1} j_{\varepsilon}^{2} d x d t
\end{aligned}
$$


which implies that $\varepsilon^{2 k} \rho_{\varepsilon}^{-\tau-2 k} j_{\varepsilon}^{2 k+1}$ converges to 0 strongly in $L_{\text {loc }}^{1}(\mathbb{R})$.

Let us set $V_{\varepsilon}=\left(p_{\varepsilon}, q_{\varepsilon}\right)$, where

$$
\begin{aligned}
& p_{\varepsilon}=-\frac{\rho_{\varepsilon}^{2 n+1}}{2 n+1}-\sum_{k=1}^{n} b_{n, k} \varepsilon^{2 k} \rho_{\varepsilon}^{2(n-k)+1} j_{\varepsilon}^{2 k}, \\
& q_{\varepsilon}=\varepsilon^{2} \sum_{k=0}^{n} a_{n, k} \varepsilon^{2 k} \rho_{\varepsilon}^{2(n-k)} j_{\varepsilon}^{2 k+1} .
\end{aligned}
$$

Then, equation (4.21) shows that $\left\{\operatorname{curl} V_{\varepsilon}\right\}$ is bounded in $L^{2}(A)$ for all $A$ as before. Since $V_{\varepsilon}$ is also bounded in $L^{2}(A)$, as can be easily checked from the definitions of $p_{\varepsilon}$ and $q_{\varepsilon}$ with the same argument leading to (4.23), we are in a position to apply the div-curl lemma 4.1, and deduce that the product $\left\langle U_{\varepsilon}, V_{\varepsilon}\right\rangle$ converges (along subsequences) in $D^{\prime}$ to $\langle U, V\rangle$, where

$$
\begin{aligned}
& U=w-\lim U_{\varepsilon}=(\rho, j), \\
& V=w-\lim V_{\varepsilon}=(p, q) .
\end{aligned}
$$

By the same bounds we used in (4.24), we deduce that

$$
\begin{gathered}
\sum_{k=1}^{n} b_{n, k} \varepsilon^{2 k} \rho_{\varepsilon}^{2(n-k)+1} j_{\varepsilon}^{2 k} \longrightarrow 0, \quad \sum_{k=1}^{n} \rho_{\varepsilon} b_{n, k} \varepsilon^{2 k} \rho_{\varepsilon}^{2(n-k)+1} j_{\varepsilon}^{2 k} \longrightarrow 0 \\
\varepsilon^{2} \sum_{k=0}^{n} a_{n, k} \varepsilon^{2 k} \rho_{\varepsilon}^{2(n-k)} j_{\varepsilon}^{2 k+1} \longrightarrow 0, \varepsilon^{2} j_{\varepsilon} \sum_{k=0}^{n} a_{n, k} \varepsilon^{2 k} \rho_{\varepsilon}^{2(n-k)} j_{\varepsilon}^{2 k+1} \longrightarrow 0
\end{gathered}
$$

strongly in $L^{1}(A)$. Hence we see that (along subsequences)

$$
p=w-\lim \left(-\frac{\rho_{\varepsilon}^{2 n+1}}{2 n+1}\right), \quad q=0,
$$

while

$$
\langle U, V\rangle=-w-\lim \left(\frac{\rho_{\varepsilon}^{2 n+2}}{2 n+1}\right)
$$

In other words, we have shown that

$$
w-\lim \rho_{\varepsilon}^{2 n+2}=\left(w-\lim \rho_{\varepsilon}^{2 n+1}\right) \rho .
$$


As is well-known in such contexts, the conclusion of Proposition 4.1 automatically holds. One possible proof consists in recalling that, by convexity,

$$
w-\lim \rho_{\varepsilon}^{2 n+2} \geq\left(w-\lim \rho_{\varepsilon}^{2 n+1}\right)^{(2 n+2) /(2 n+1)},
$$

so that

$$
w-\lim \rho_{\varepsilon}^{2 n+2} \geq \rho^{2 n+2} .
$$

Inequality (4.30), combined with (4.29) yields that $w-\lim \rho_{\varepsilon}^{2 n+2}=$ $\rho^{2 n+2}$.

We may also use Minty's trick, as in [Lio], [MaMi] to conclude that $w-\lim \rho_{\varepsilon}^{2 n+1}=\rho^{2 n+1}$. In both cases, we deduce the strong convergence of $\rho_{\varepsilon}$ to $\rho$ using the strict convexity of $f^{m}(t), t \in[0, \infty)$, for $m \geq 1$.

We are now able to handle the singular case.

Theorem 4.3. Let $\alpha<-1$, and let $\left(\rho_{\varepsilon}, j_{\varepsilon}\right)$ be a sequence of solutions to the initial-boundary value problem for the system (4.2), where the initial values $u_{0}^{\varepsilon}, v_{0}^{\varepsilon}$ for the kinetic system (1.5) satisfy the hypotheses of Theorem 3.1, part iii). Then, there exists $\rho \in L_{\mathrm{loc}}^{1} \cap L^{\infty}$ such that $\rho_{\varepsilon}(x, t)$ converges to $\rho(x, t)$ in $L_{\mathrm{loc}}^{p}(\mathbb{R} \times(0, T])$ for all $p \in[1,+\infty)$ and all $T>0$, while $\varepsilon j_{\varepsilon}(x, t)$ converges to zero strongly in $L_{\mathrm{loc}}^{2}(\mathbb{R} \times[0, T])$. The limit density $\rho(x, t)$ is the weak solution to the Cauchy problem for the porous media equation in $D^{\prime}(\mathbb{R} \times(0, T])$, with initial datum $\rho_{0}$ that is the weak limit of $\rho_{\varepsilon}(x, t=0)=u_{0}^{\varepsilon}+v_{0}^{\varepsilon}$.

Proof. Let us rewrite the second equation of the system (4.2) in the equivalent form (4.21), where $n$ has been chosen in such a way that $\tau$ satisfies the hypotheses of Theorem (3.1) (bound (3.27)), and at the same time $\tau>1$. Let us remark that this is always possible in view of (4.17) and (4.20). Let

$$
v_{\varepsilon}=-2\left(\rho_{\varepsilon}^{-\tau} j_{\varepsilon}+\sum_{k=1}^{n} c_{n, k} \varepsilon^{2 k} \rho_{\varepsilon}^{-\tau-2 k} j_{\varepsilon}^{2 k+1}\right) .
$$

By the proof of Proposition 4.1 we see that the sequence $\left\{v_{\varepsilon}\right\}$ has a weak limit in $L^{2}$, and that

$$
v=w-\lim v_{\varepsilon}=w-\lim \left(-2 \rho_{\varepsilon}^{-\tau} j_{\varepsilon}\right) .
$$


Using the definitions (4.25), (4.21) becomes

$$
\frac{\partial q_{\varepsilon}}{\partial t}-\frac{\partial p_{\varepsilon}}{\partial x}=v_{\varepsilon}
$$

We pass to the limit in the sense of distributions in (4.32), and recall that $q_{\varepsilon}$ converges to zero in $D$. We find

$$
v=\frac{\partial}{\partial x} \frac{\rho^{2 n+1}}{2 n+1}
$$

in $D^{\prime}$. However, since $v \in L^{2}, \partial \rho^{2 n+1} / \partial x \in L^{2}$ as well. Let us write now the continuity equation as

$$
\frac{\partial \rho_{\varepsilon}}{\partial t}-\frac{\partial}{\partial x}\left(\frac{\rho_{\varepsilon}^{\tau} v_{\varepsilon}}{2}+\sum_{k=1}^{n} c_{n, k} \varepsilon^{2 k} \rho_{\varepsilon}^{-2 k} j_{\varepsilon}^{2 k+1}\right)=0
$$

Since $\rho_{\varepsilon} \longrightarrow \rho$ in $L_{\text {loc }}^{p}$ strongly for $p \in[1,+\infty)$, and $\rho_{\varepsilon}$ is bounded in $L^{\infty}$, we have

$$
\rho_{\varepsilon}^{\tau} v_{\varepsilon} \longrightarrow \rho^{\tau} v=\left(\rho^{2 n+1}\right)^{\tau /(2 n+1)} \frac{\partial}{\partial x} \frac{\rho^{2 n+1}}{2 n+1} \frac{\partial}{\partial x} \frac{\rho^{1+|\alpha|}}{1+|\alpha|}
$$

in $L^{2}$-weak. Consequently, as $\varepsilon \longrightarrow 0$, for all $\phi \in C^{\infty}$, such that $\operatorname{supp} \phi \subset \mathbb{R} \times \mathbb{R}^{+}$

$$
\int_{0}^{+\infty} \int_{-\infty}^{+\infty}\left(\phi_{t} \rho-\phi_{x} \frac{\rho^{1+|\alpha|}}{1+|\alpha|}\right) d x d t+\int_{-\infty}^{+\infty} \phi(x, 0) \rho(x, 0) d x
$$

The Cauchy problem for the porous media equation, with initial data $\rho_{0}$ satisfying the hypotheses of the theorem, is well-posed in the weak sense (4.36). In fact, existence, uniqueness and continuous dependence on the data for (4.34) is known ( $c f$. Aronson [Aro]). The existence theorem guarantees a unique limit to the singular perturbation problem (4.2).

\section{Extension to higher dimensions.}

In this section, we shall discuss the three dimensional model (1.7). In fact, all the results can be adapted to an arbitrary number of dimensions, and we just choose to emphasize the three dimensional example. 
Moreover, the largest part of the one-dimensional arguments can be adapted to higher dimensions, so we just sketch the main differences.

We shall study equations $(1.8)$ in the box $\Omega=(-a, a)^{3}$ with periodic boundary conditions. Besides, we will limit our analysis to the case of a rate function $k$ of the type $\rho^{\alpha}$, with $\alpha<1$. In analogy with the one-dimensional model, we will also write system (1.7) in the equivalent form

$$
\left\{\begin{array}{l}
\frac{\partial u^{ \pm}}{\partial t} \pm \frac{1}{\varepsilon} \frac{\partial u^{ \pm}}{\partial x}=\frac{1}{\varepsilon^{2}} \rho^{\alpha}\left(\rho-6 u^{ \pm}\right), \\
\frac{\partial v^{ \pm}}{\partial t} \pm \frac{1}{\varepsilon} \frac{\partial v^{ \pm}}{\partial y}=\frac{1}{\varepsilon^{2}} \rho^{\alpha}\left(\rho-6 v^{ \pm}\right) \\
\frac{\partial w^{ \pm}}{\partial t} \pm \frac{1}{\varepsilon} \frac{\partial w^{ \pm}}{\partial z}=\frac{1}{\varepsilon^{2}} \rho^{\alpha}\left(\rho-6 w^{ \pm}\right),
\end{array}\right.
$$

where $u=\left(u^{+}, u^{-}, v^{+}, v^{-}, w^{+}, w^{-}\right)=\left(u_{1}, u_{4}, u_{2}, u_{5}, u_{3}, u_{6}\right)$.

As in the one-dimensional case, given any convex function $\varphi(r)$, $r \geq 0$, we deduce the estimate

$$
\begin{aligned}
& \int_{\Omega} \sum_{i=1}^{6} \frac{\partial \varphi\left(u_{i}\right)}{\partial t} d x \\
& \quad=-\frac{1}{2 \varepsilon^{2}} \int_{\Omega} \rho^{\alpha} \sum_{i \neq j}\left(\varphi^{\prime}\left(u_{i}\right)-\varphi^{\prime}\left(u_{j}\right)\right)\left(u_{i}-u_{j}\right) d x \\
& \leq 0 .
\end{aligned}
$$

In particular, if $\varphi(r)=r^{p}, p>1$, we obtain

$$
\int_{\Omega} \frac{\partial}{\partial t} \sum_{i=1}^{6} u_{i}^{p} d x=-\frac{p}{2 \varepsilon^{2}} \int_{\Omega} \rho^{\alpha} \sum_{i \neq j}\left(u_{i}-u_{j}\right)\left(u_{i}^{p-1}-u_{j}^{p-1}\right) d x
$$

The existence theory follows as in the one-dimensional case, and we obtain the corresponding of Propositions 2.1 and 2.2.

Proposition 5.1. Let $0 \leq \alpha<1$, and let $0 \leq u_{0, j}(x) \in L^{\infty}(\Omega)$, $j=1, \ldots, 6$. Then, the initial-boundary value problem for system (5.1) has a unique solution $u(x, t)$, such that, for $j=1, \ldots, 6, u_{j}(x, t) \in$ $L^{\infty}(\Omega \times(0, T)) \cap C\left([0, T] ; L^{p}(\Omega)\right)$ for all $T>0$ and $1 \leq p<\infty$. In addition, the solution satisfies the following bound

$$
\max _{j \leq 6}\left\|u_{j}\left(\cdot, t_{2}\right)\right\|_{\infty} \leq \max _{j \leq 6}\left\|u_{j}\left(\cdot, t_{1}\right)\right\|_{\infty}, \quad \text { if } t_{1} \leq t_{2} .
$$


Proposition 5.2. Let $\alpha<0$, and let $u_{0, j}(x) \in L^{\infty}(\Omega)$ satisfy the lower bound $u_{0, j} \geq \delta$ on $\Omega$ for some $\delta>0$. Then, the initial-boundary value problem for the system (5.1) has a unique solution bounded away from zero $u(x, t)$ such that for $j=1, \ldots, 6, u_{j}(x, t) \in L^{\infty}(\Omega \times(0, T)) \cap$ $C\left([0, T] ; L^{p}(\Omega)\right)$ for all $T>0$ and $1 \leq p<\infty$. In addition, this solution satisfies the bounds (5.4) and

$$
\inf _{\substack{x \in \Omega \\ j \leq 6}} u_{j}\left(x, t_{1}\right) \leq \inf _{\substack{x \in \Omega \\ j \leq 6}} u_{j}\left(x, t_{2}\right), \quad \text { if } t_{1} \leq t_{2}
$$

Given $u=\left(u_{1}, \ldots, u_{6}\right)$, let us now introduce the operator $B^{\alpha}$ defined by components by

$$
\begin{aligned}
B_{j}^{\alpha} u=-\frac{\partial u_{j}}{\partial x_{j}}+\rho^{\alpha}\left(\rho-6 u_{j}\right), & i=1,2,3, \\
B_{j}^{\alpha} v=\frac{\partial u_{j}}{\partial x_{j}}+\rho^{\alpha}\left(\rho-6 u_{j}\right), & i=4,5,6 .
\end{aligned}
$$

Then, the following lemma is immediate

Lemma 5.1. Let $0 \leq \alpha \leq 1 / 5$. Then, the operator $B^{\alpha}$ is dissipative from the domain

$$
D^{+}\left(B^{\alpha}\right)=\left\{u \in\left[W^{1,1}(\Omega)\right]^{6}\right\}
$$

into $\left[L^{1}(\Omega)\right]^{6}$.

If $-1 \leq \alpha<0$, and if $\delta>0$, the operator $B_{\alpha}$ is dissipative from the domain

$$
D_{\delta}^{+}\left(B^{\alpha}\right)=\left\{u \in\left[W^{1,1}(\Omega)\right]^{6}, u_{j} \geq \delta, j=1, \ldots, 6\right\}
$$

into $\left[L^{1}(\Omega)\right]^{6}$.

Thus, we obtain the analogue of Proposition 2.3.

Proposition 5.3. Let $0 \leq u_{0, j}(x) \in L^{1}(\Omega) \cap L^{\infty}(\Omega), j=1, \ldots, 6$. Then, provided $-1 \leq \alpha \leq 1 / 5$, the initial-boundary value problem (5.1) has a unique global solution $u(x, t)$ such that $u_{j}(x, t) \in L^{\infty}(\Omega \times(0, T)) \cap$ 
$C\left([0, T] ; L^{1}(\Omega) \cap L^{\infty}(\Omega)\right)$ for all $T \geq 0$ and $j=1, \ldots, 6$. Moreover, for all $t \geq 0$

$$
\sum_{j=1}^{6}\left\|u_{j}(x+h, t)-u_{j}(x, t)\right\|_{1} \leq \sum_{j=1}^{6}\left\|u_{0, j}(x+h)-u_{0, j}(x)\right\|_{1} .
$$

If $0 \leq \alpha \leq 1 / 5$, these results extend to $\Omega=\mathbb{R}^{3}$.

As in Section 3, we can obtain entropy bounds, that allow us to pass to the limit. Let us briefly outline the main differences. We introduce functions $0 \leq u_{0, j} \in L^{1}(\mathbb{R}) \cap L^{\infty}(\mathbb{R}), j=1, \ldots, 6$ such that

$$
\int_{\mathbb{R}^{3}} \sum_{j=1}^{6} u_{0, j}(x)\left|\log u_{0, j}(x)\right| d x=M_{1}<\infty
$$

and, for $\omega(x)=\left(1+x^{2}\right)^{\beta}, 0<\beta<1 / 8$,

$$
\int_{\mathbb{R}^{3}} \omega(x) \sum_{j=1}^{6} u_{0, j}^{2}(x) d x=M_{2}<\infty .
$$

Given $\gamma>0$, let us denote with $\Omega_{\varepsilon}$, the domain $\left(-1 / \varepsilon^{\gamma}, 1 / \varepsilon^{\gamma}\right)^{3}$. In addition, given $\mu>0$, let for $j=1, \ldots, 6$

$$
u_{0, j}^{\varepsilon}=\max \left\{u_{0}(x), \varepsilon^{\mu}\right\} .
$$

By Proposition 5.2, if $\alpha<0$, the initial boundary value problem for the system (5.1), with periodic boundary conditions on $\Omega_{\varepsilon}$, and initial values (5.9), has a unique global solution $u^{\varepsilon}(x, t)$.

Moreover, provided $\mu>\gamma(3+\beta), u_{0}^{\varepsilon}$ satisfies bounds (5.7) and (5.8) with different but finite constants $M_{1}^{\varepsilon}$ and $M_{2}^{\varepsilon}$.

The proof of the entropy bounds follows along the same lines of Section 3. We only remark that, in consequence of (5.8), one has to study the time evolution of

$$
\int_{\Omega_{\varepsilon}}\left(\sum_{j} u_{j}^{\varepsilon}(x, t) \log u_{j}^{\varepsilon}(x, t)+\omega(x) \sum_{j}\left(u_{j}^{\varepsilon}(x, t)\right)^{2}\right) d x .
$$

Since the integrand on the right-hand side of (5.3) is nonpositive for any convex function $\varphi(r)$, choosing $\varphi(r)=r^{2}$ we obtain

$$
\begin{aligned}
\frac{d}{d t} \int_{\Omega_{\varepsilon}} \omega(x) \sum_{j}\left(u_{j}^{\varepsilon}(x, t)\right)^{2} d x & \\
& -\frac{1}{\varepsilon} \sum_{j=1}^{3} \int_{\Omega_{\varepsilon}} \omega(x) \frac{\partial}{\partial x_{j}}\left(\left(u_{j}^{\varepsilon}\right)^{2}-\left(u_{j+3}^{\varepsilon}\right)^{2}\right) d x \leq 0 .
\end{aligned}
$$


Hence, integrating by parts and making use of the periodicity, we deduce the analogous of (3.7)

$$
\begin{aligned}
\frac{d}{d t} \int_{\Omega_{\varepsilon}} \omega(x) \sum_{j}\left(u_{j}^{\varepsilon}(x, t)\right)^{2} d x & \\
& -\frac{1}{\varepsilon} \sum_{j=1}^{3} \int_{\Omega_{\varepsilon}} \frac{\partial}{\partial x_{j}} \omega(x)\left(\left(u_{j}^{\varepsilon}\right)^{2}-\left(u_{j+3}^{\varepsilon}\right)^{2}\right) d x \leq 0 .
\end{aligned}
$$

We next observe that $\left(\partial / \partial x_{j}\right) \omega \in L^{4}\left(\mathbb{R}^{3}\right), j=1,2,3$. This implies the analogous of (3.14)

A further step consists in the identification of the limit Maxwellian. Let us set $p=2$ in (5.3). Then, integrating over time we get

$$
\frac{1}{\varepsilon^{2}} \int_{0}^{T} \int_{\Omega_{\varepsilon}} \rho_{\varepsilon}^{\alpha} \sum_{i \neq j}\left(u_{i}^{\varepsilon}-u_{j}^{\varepsilon}\right)^{2} d x d t \leq \int_{\Omega_{\varepsilon}} \sum_{i} u_{0, i}^{2} d x
$$

Now, considering that the solution satisfies the bound (5.4), if $\alpha<0$,

$$
\rho_{\varepsilon}^{\alpha} \geq\left(6 \max _{i \leq 6}\left\|u_{0, i}\right\|_{\infty}\right)^{\alpha}=\nu^{\alpha}
$$

and by (5.10) we obtain

$$
\frac{\nu^{\alpha}}{\varepsilon^{2}} \int_{0}^{T} \int_{\Omega_{\varepsilon}} \sum_{i \neq j}\left(u_{i}^{\varepsilon}-u_{j}^{\varepsilon}\right)^{2} d x d t \leq \int_{\Omega_{\varepsilon}} \sum_{i} u_{0, i}^{2} d x .
$$

Inequality (5.12) implies that, for $i \neq j, u_{i}^{\varepsilon}-u_{j}^{\varepsilon} \longrightarrow 0$ strongly in $L^{2}\left([0, T] \times \Omega_{\varepsilon}\right)$.

The same conclusion can be derived when $0<\alpha<1$. Since in this case (5.11) does not hold, we shall make use of a different argument. Let us write (5.3) by taking $p=2-\alpha$. We obtain

$$
\begin{aligned}
\frac{2-\alpha}{2 \varepsilon^{2}} \int_{0}^{T} \int_{\mathbb{R}^{3}} \rho_{\varepsilon}^{\alpha} \sum_{i \neq j}\left(u_{i}^{\varepsilon}-u_{j}^{\varepsilon}\right)\left(\left(u_{i}^{\varepsilon}\right)^{1-\alpha}-\left(u_{j}^{\varepsilon}\right)^{1-\alpha}\right) d x d t & \\
& \leq \int_{\mathbb{R}^{3}} \sum_{i=1}^{6} u_{0, i}^{2-\alpha} d x .
\end{aligned}
$$


This implies

$$
(1-\alpha) \int_{0}^{T} \int_{\mathbb{R}^{3}} \sum_{i \neq j}\left(u_{i}^{\varepsilon}-u_{j}^{\varepsilon}\right)^{2} d x d t \leq \frac{\varepsilon^{2}}{2-\alpha} \int_{\mathbb{R}^{3}} \sum_{i} u_{0, i}^{2-\alpha} d x
$$

and also in this case, $u_{i}^{\varepsilon}-u_{j}^{\varepsilon} \rightarrow 0$ strongly in $L^{2}\left([0, T] \times \mathbb{R}^{3}\right)$ for $i \neq j$.

The rest of the proof leading to Theorem 3.1 follows along the same lines of the one-dimensional situation. So we have

Theorem 5.1. Let $0 \leq u_{0, j} \in L^{1}\left(\mathbb{R}^{3}\right) \cap L^{\infty}\left(\mathbb{R}^{3}\right), j=1, \ldots, 6$ satisfy conditions (5.7) and (5.8).

i) Let $0 \leq \alpha<1$. Then, for all $T>0$, and $\varepsilon>0$ there exist constants $d_{1}=d_{1}\left(u_{0}, T\right), d_{2}=d_{2}\left(u_{0}, T\right)$ and $d_{3}=d_{3}\left(u_{0}, T\right)$ such that, the unique solution $u^{\varepsilon}(x, t)$ to the initial value problem for system (5.1) satisfies

$$
\begin{gathered}
\int_{0}^{T} \int_{\mathbb{R}^{3}}\left(\frac{u_{i}^{\varepsilon}(x, t)-u_{i+3}^{\varepsilon}(x, t)}{\varepsilon}\right)^{2} d x d t \leq d_{1} \\
\int_{0}^{T} \int_{\mathbb{R}^{3}}\left(\frac{u_{i}^{\varepsilon}(x, t)-u_{i+3}^{\varepsilon}(x, t)}{\varepsilon}\right)^{2} \rho_{\varepsilon}(x, t)^{\alpha-1} d x d t \leq d_{2}
\end{gathered}
$$

where, in both cases, $i=1,2,3$, and

$$
\begin{gathered}
\int_{\mathbb{R}^{3}}\left(\sum_{i} u_{i}^{\varepsilon}(t)\left|\log u_{i}^{\varepsilon}(t)\right|+\omega \sum_{i}\left(u_{i}^{\varepsilon}(t)\right)^{2}\right) d x \leq d_{3}, \\
\lim _{\varepsilon \rightarrow 0} \int_{0}^{T} \int_{\mathbb{R}^{3}} \sum_{i \neq j}\left(u_{i}^{\varepsilon}-u_{j}^{\varepsilon}\right)^{2} d x d t=0 .
\end{gathered}
$$

ii) Let $-1 \leq \alpha<0$. Then, for all $T>0$, and $k \in \mathbb{N}^{+}$, the unique solution $u^{\varepsilon}(x, t)$ to the initial-boundary value problem for system (5.1) on the domain $\Omega_{\varepsilon}=\left(-1 / \varepsilon^{k}, 1 / \varepsilon^{k}\right)^{3}$ satisfies the bounds (5.15), (5.16), (5.17) and the property (5.18) in $[0, T] \times \Omega_{\varepsilon}$.

iii) Let $\alpha<-1$. Given $k \in \mathbb{N}^{+}$, let $\mu>k(3+\beta)$, and let $u_{0}^{\varepsilon}$ be defined by (5.9). Then, for all $T>0$, the unique solution to the initial-boundary value problem for system (5.1) on the domain $\Omega_{\varepsilon}=$ 
$\left(-1 / \varepsilon^{k}, 1 / \varepsilon^{k}\right)^{3}$, with initial data $u_{0}^{\varepsilon}$, satisfies the bounds (5.10), (5.15), (5.17) and the property (5.18) in $\Omega_{\varepsilon}$. In addition we have

$$
\int_{0}^{T} \int_{\Omega_{\varepsilon}}\left(\frac{u_{i}^{\varepsilon}(x, t)-u_{i+3}^{\varepsilon}(x, t)}{\varepsilon}\right)^{2} \rho_{\varepsilon}(x, t)^{-2 \tau} d x d t \leq d_{2},
$$

where $i=1,2,3$, for all $0<\tau \leq(|\alpha|+1) / 2$.

Let us denote by $j_{\varepsilon}(x, t)$ the flux, i.e.

$$
j_{\varepsilon}=\left(\frac{u_{\varepsilon}^{+}-u_{\varepsilon}^{-}}{\varepsilon}, \frac{v_{\varepsilon}^{+}-v_{\varepsilon}^{-}}{\varepsilon}, \frac{w_{\varepsilon}^{+}-w_{\varepsilon}^{-}}{\varepsilon}\right) .
$$

Then, the macroscopic equations for the system (5.1) can be expressed in the form

$$
\left\{\begin{array}{l}
\frac{\partial \rho_{\varepsilon}}{\partial t}+\operatorname{div} j_{\varepsilon}=0, \\
\varepsilon^{2} \frac{\partial j_{\varepsilon}}{\partial t}+\frac{1}{3} \operatorname{grad} \rho_{\varepsilon}=-6 \rho_{\varepsilon}^{\alpha} j_{\varepsilon}+E_{\varepsilon}
\end{array}\right.
$$

where $E_{\varepsilon}$ denotes the vector

$$
\begin{aligned}
E_{\varepsilon}= & \frac{\partial}{\partial x}\left(\frac{1}{3} \rho_{\varepsilon}-\left(u_{\varepsilon}^{+}-u_{\varepsilon}^{-}\right)\right) \\
& \left.\frac{\partial}{\partial y}\left(\frac{1}{3} \rho_{\varepsilon}-\left(v_{\varepsilon}^{+}-v_{\varepsilon}^{-}\right)\right), \frac{\partial}{\partial z}\left(\frac{1}{3} \rho_{\varepsilon}-\left(w_{\varepsilon}^{+}-w_{\varepsilon}^{-}\right)\right)\right) .
\end{aligned}
$$

The presence of the vector $E_{\varepsilon}$ is the main difference between the onedimensional system (4.2) and the three-dimensional system (5.21). This is a consequence of the fact that in more than one-dimension the system of the macroscopic equations can not be expressed only in terms of the mean quantities. Let us rewrite

$$
E_{\varepsilon}=\left(\frac{\partial}{\partial x} e_{\varepsilon}^{1}, \frac{\partial}{\partial y} e_{\varepsilon}^{2}, \frac{\partial}{\partial z} e_{\varepsilon}^{3}\right)
$$

with obvious meaning of $e_{\varepsilon}^{1}, e_{\varepsilon}^{2}$, and $e_{\varepsilon}^{3}$. We have

$(5.23) e_{\varepsilon}^{1}=\frac{1}{3}\left(v_{\varepsilon}^{+}-u_{\varepsilon}^{-}\right)+\frac{1}{3}\left(v_{\varepsilon}^{-}-u_{\varepsilon}^{-}\right)+\frac{1}{3}\left(w_{\varepsilon}^{+}-u_{\varepsilon}^{+}\right)+\frac{1}{3}\left(w_{\varepsilon}^{-}-u_{\varepsilon}^{-}\right)$, 
so that, thanks to (5.18) we conclude that $e_{\varepsilon}^{1}$ converges to zero strongly in $L_{x, t}^{2}$. The same conclusion holds for the other components. We are in a position to obtain the corresponding of Theorems 4.1 and 4.2, provided $-1 \leq \alpha \leq 1 / 5$. In this range of $\alpha$, by dissipativity, we have the compactness of $\rho_{\varepsilon}(x, t)$ in $L_{x}^{1}$ or $L_{\text {loc }}^{1}$ if $\alpha<0$. Let us remark that when $\alpha$ is positive, $1 / 5<\alpha<1$ we can derive the same result of compactness as a consequence of the "div-curl" Lemma 4.1. Let us set $U_{\varepsilon}=\left(j_{\varepsilon}, \rho_{\varepsilon}\right)$ and $V_{\varepsilon}=\left(0, \rho_{\varepsilon}\right)$. Then, the first of equations (5.21) reads $\operatorname{div} U_{\varepsilon}=0$, and this obviously implies that $\operatorname{div} U_{\varepsilon}$ is bounded in $L_{x, t}^{2}$. In view of (5.15) we deduce that $\varepsilon^{2} j_{\varepsilon}$ converges to zero strongly in $L_{x, t}^{2}$, and by (5.16) follows that $\rho_{\varepsilon}^{\alpha} j_{\varepsilon}$ is bounded in $L_{x, t}^{2}$. But $e_{\varepsilon}^{1}, e_{\varepsilon}^{2}$, and $e_{\varepsilon}^{3}$ converge to zero strongly in $L_{x, t}^{2}$. Consequently, from the second equation of the system (5.21) we obtain that $\operatorname{curl} V_{\varepsilon}$ is compact in $H_{x, t}^{-1}$, and $U_{\varepsilon} V_{\varepsilon}=\rho_{\varepsilon}^{2}$ passes to the limit. At this point, we deduce as in Section 4 that $\rho_{\varepsilon}(x, t)$ converges to $\rho(x, t)$ in $L_{\mathrm{loc}}^{p}, 1 \leq p<\infty$. Finally, thanks to the bound (5.17), we can show that

$$
\lim _{R \rightarrow \infty} \sup _{0 \leq t \leq T} \int_{|x|>R} \rho_{\varepsilon}(x, t) d x=0
$$

and, since $\rho_{\varepsilon}$ is bounded in $L^{\infty}, \rho_{\varepsilon}$ converges to $\rho$ in $L^{p}(\mathbb{R})^{3}$. So we have

Theorem 5.2. Let $0 \leq \alpha<1$, and let $\left(\rho_{\varepsilon}, j_{\varepsilon}\right)$ be a sequence of solutions to the initial value problem for the system (5.21), where the initial values $u_{0, j}, j=1, \ldots, 6$ satisfy the hypotheses of Proposition 5.1 and Theorem 5.1. Then, there exists $\rho \in L^{1} \cap L^{\infty}$ such that $\rho_{\varepsilon}(x, t)$ converges to $\rho(x, t)$ strongly in $C\left([0, T] ; L^{p}\left(\mathbb{R}^{3}\right)\right)$ for all $T \geq 0$ and $1 \leq p<\infty$, while $\varepsilon j_{\varepsilon}$ converges to zero strongly in $L^{2}\left(\mathbb{R}^{3} \times[0, T]\right)$. The limit density $\rho(x, t)$ is the (unique) weak solution to the Cauchy problem for the fast diffusion equation

$$
\frac{\partial \rho}{\partial t}-\frac{1}{18(1-\alpha)} \Delta \rho^{1-\alpha}=0, \quad \text { in } D^{\prime}\left(\mathbb{R}^{3} \times(0, \infty)\right),
$$

with initial datum $\rho_{0}=\sum_{j=1}^{6} u_{0, j}$.

Theorem 5.3. Let $-1 \leq \alpha<0$, and let $\left(\rho_{\varepsilon}, j_{\varepsilon}\right)$ be a sequence of solutions to the initial-boundary value problem for the system (5.21), where the initial values $u_{0, j}, j=1, \ldots, 6$ satisfy the hypotheses of Proposition 5.3 and Theorem 5.1. Then, there exists $\rho \in L_{\mathrm{loc}}^{1} \cap L^{\infty}$ such that $\rho_{\varepsilon}(x, t)$ 
converges to $\rho(x, t)$ in $C\left([0, T] ; L_{\mathrm{loc}}^{p}\left(\mathbb{R}^{3}\right)\right)$ for all $T \geq 0$ and $p \in[1, \infty)$, while $\varepsilon j_{\varepsilon}(x, t)$ converges to zero strongly in $L_{\text {loc }}^{2}\left(\mathbb{R}^{3} \times[0, T]\right)$. The limit density $\rho(x, t)$ is the (unique) weak solution to the Cauchy problem for the slow diffusion equation

$$
\frac{\partial \rho}{\partial t}-\frac{1}{18(1+|\alpha|)} \Delta \rho^{1+|\alpha|}=0, \quad \text { in } D^{\prime}\left(\mathbb{R}^{3} \times(0, \infty)\right),
$$

with initial datum $\rho_{0}=\sum_{j=1}^{6} u_{0, j}$.

REMARK 5.1. As in Section 4 we observe that the $u_{j}^{\varepsilon}(x, t), j=1,2, \ldots$, 6 , solutions to the initial (if $0 \leq \alpha<1$ ) (or initial-boundary, if $-1 \leq$ $\alpha<0$ ) value problem for the kinetic system (5.1) converge strongly to $\rho / 6$, where $\rho$ is the solution of the corresponding nonlinear diffusion equation.

We will now examine the case $\alpha<-1$. Let $\rho_{1, \varepsilon}=u_{\varepsilon}^{+}+u_{\varepsilon}^{-}, \rho_{2, \varepsilon}=$ $v_{\varepsilon}^{+}+v_{\varepsilon}^{-}, \rho_{3, \varepsilon}=w_{\varepsilon}^{+}+w_{\varepsilon}^{-}$, and let $j_{i, \varepsilon}, i=1,2,3$ be the components of $j_{\varepsilon}$, given by (5.20). Then, summing and subtracting the first two equations (5.1), we obtain the system

$$
\left\{\begin{array}{l}
\frac{\partial \rho_{1, \varepsilon}}{\partial t}+\frac{\partial j_{1, \varepsilon}}{\partial x}=\frac{2}{\varepsilon^{2}} \rho_{\varepsilon}^{\alpha}\left(\rho_{\varepsilon}-3 \rho_{1, \varepsilon}\right), \\
\varepsilon^{2} \frac{\partial j_{1, \varepsilon}}{\partial t}+\frac{\partial \rho_{1, \varepsilon}}{\partial x}=-2 \rho_{\varepsilon}^{\alpha} j_{1, \varepsilon}, \quad x \in \mathbb{R}, t \geq 0 .
\end{array}\right.
$$

The analysis of Section 4, following Lemma 4.1 can easily be applied to system (5.25). With few differences, due to the presence of the term on the right side of the first equation, we will arrive to analogous conclusions. In particular, since $\rho_{\varepsilon}$ is bounded in $L^{\infty}$, and, in view of (5.19) $\rho_{\varepsilon}^{-(|\alpha|+1) / 2} j_{1, \varepsilon}$ is bounded in $L_{x, t}^{2}, \rho_{\varepsilon}^{\alpha} \rho_{1, \varepsilon} j_{1, \varepsilon}$ is bounded in $L_{x, t}^{2}$ provided $\alpha \geq-3$. The same argument shows that $j_{1, \varepsilon} \rho_{\varepsilon}^{\alpha}\left(\rho_{\varepsilon}-3 \rho_{1, \varepsilon}\right) / \varepsilon^{2}$ is bounded in $L_{x, t}^{2}$ if $\alpha \geq-3$. Hence, $(\partial / \partial x) \rho_{1, \varepsilon}^{2}$ is compact in $H_{x, t}^{-1}$.

By identical computations, summing and subtracting the third and fourth (respectively the fifth and sixth) equation (5.1), we deduce that, if $\alpha \geq-3$, both $(\partial / \partial y) \rho_{2, \varepsilon}^{2}$ and $(\partial / \partial z) \rho_{3, \varepsilon}^{2}$ are compact in $H_{x, t}^{-1}$. Moreover, in view of (5.18), $\rho_{i, \varepsilon}^{2}-\rho_{j, \varepsilon}^{2}$ converges to zero in $L_{x, t}^{2}$. So we conclude that $\operatorname{grad} \rho_{\varepsilon}^{2}$ is compact in $H_{x, t}^{-1}$.

This result, coupled with the first equation of the system (5.21) enables us to handle, by the "div-curl" lemma, the passage to the limit for $-3 \leq \alpha<-1$, exactly as in the one-dimensional case. 
The extension to $\alpha<-3$ follows by the same strategy we adopted above, along the same lines of the one-dimensional proof, first multiplying the second equation of the system (5.25) by $\rho_{1, \varepsilon}^{n}, n>1$, and then recovering the equivalent system in which the terms on the right-hand sides are bounded in $L_{x, t}^{2}$.

Finally we prove

Theorem 5.4. Let $\alpha<-1$, and let $\left(\rho_{\varepsilon}, j_{\varepsilon}\right)$ be a sequence of solutions to the initial-boundary value problem for the system (5.21), where the initial values $u_{0, j}^{\varepsilon}, j=1, \ldots, 6$ for the kinetic system (5.1) satisfy the hypotheses of Theorem 5.1, part iii). Then, there exists $\rho \in L_{\mathrm{loc}}^{1} \cap L^{\infty}$ such that $\rho_{\varepsilon}(x, t)$ converges to $\rho(x, t)$ in $L_{\mathrm{loc}}^{p}\left(\mathbb{R}^{3} \times(0, T]\right)$ for all $p \in$ $[1,+\infty)$ and all $T>0$, while $\varepsilon j_{\varepsilon}(x, t)$ converges to zero strongly in $L_{\mathrm{loc}}^{2}(\mathbb{R} \times[0, T])$. The limit density $\rho(x, t)$ is the weak solution to the Cauchy problem for the porous media equation

$$
\frac{\partial \rho}{\partial t}-\frac{1}{18(1+|\alpha|)} \Delta \rho^{1+|\alpha|}=0
$$

in $D^{\prime}\left(\mathbb{R}^{3} \times(0, T]\right)$, with initial datum $\rho_{0}$ that is the weak limit of $\rho_{\varepsilon}(x, t=0)=\sum_{j=1}^{6} u_{0, j}^{\varepsilon}$.

\section{Variants and extensions.}

In this section, we present briefly a few variants and extensions of the previous problems and results. For most of these variants and extensions the proofs are straightforward adaptations of the proofs introduced above. First of all, we can allow the rate function $k$ to depend on $x$, satisfying for instance

$$
k_{1}(x) \rho^{\alpha} \leq k(x, \rho) \leq k_{2}(x) \rho^{\beta}, \quad \text { for } 0<\rho \leq 1,
$$

where $k_{1}, k_{2} \in L^{\infty}(\mathbb{R}), \inf _{x \in \mathbb{R}} k_{1}>0$, and $\alpha, \beta<1$.

Another variant consists in replacing the right-hand side in (1.1), $k(v-u)$ (respectively, $k(u-v)$ ), by $\varphi(v)-\varphi(u)$ (respectively, $\varphi(u)-$ $\varphi(v))$, where $\varphi$ is increasing.

Next, we can treat the system in which the velocities in the streaming terms are $1 / \varepsilon+a$ and $1 / \varepsilon+b$, where $a \neq b \in \mathbb{R}$. In this case we obtain at the limit the additional presence of a linear term. 
Finally we can study the system

$$
\left\{\begin{array}{l}
\frac{\partial u}{\partial t}+\frac{1}{\varepsilon} \frac{\partial u^{m}}{\partial x}=\frac{1}{\varepsilon^{2}} \rho^{\alpha}(v-u) \\
\frac{\partial v}{\partial t}-\frac{1}{\varepsilon} \frac{\partial v^{m}}{\partial x}=\frac{1}{\varepsilon^{2}} \rho^{\alpha}(u-v)
\end{array}\right.
$$

where $m>\alpha$, which yields in the limit

$$
\frac{\partial \rho}{\partial t}-c \frac{\partial^{2}}{\partial x^{2}} \rho^{m-\alpha}=0
$$

where $c=m\left(2^{m}(m-\alpha)\right)^{-1}$. Of course, (6.2) is a weakly coupled system of one-dimensional hyperbolic scalar conservation laws, and $u, v$ are entropy solutions of (6.2).

To end this section, we now wish to look at different models involving velocity sets which are not finite anymore. We begin with a model arising in radiative transfer theory, recently studied by C. Bardos, F. Golse, B. Perthame and R. Sentis [BGPS]. This equation describes the transport of photons in a starlike medium and is, mathematically, a nonlinear version of the transport of neutrons. We look for $u_{\varepsilon}=u_{\varepsilon}(x, \omega, t) \geq 0$, where $x \in \mathbb{R}^{N}, \omega \in S^{N-1}$ (the unit sphere of $\mathbb{R}^{N}$ ), $t \geq 0$, solution of the initial (or initial-boundary) value problem for the equation

$$
\frac{\partial u}{\partial t}+\frac{1}{\varepsilon} \omega \cdot \operatorname{grad} u+\frac{1}{\varepsilon^{2}} k\left(\rho_{\varepsilon}\right)\left(u_{\varepsilon}-\rho_{\varepsilon}\right)=0 .
$$

In $(6.4) \rho_{\varepsilon}$ denotes the integral

$$
\rho_{\varepsilon}(x, t)=\int_{S^{N-1}} u_{\varepsilon}(x, \omega, t) d \omega
$$

where $d \omega$ is the normalized Lebesgue measure on $S^{N-1}\left(\int_{S^{N-1}} d \omega=1\right)$. The nonnegative function $k$ is continuous on $[0, \infty)$ and is supposed to satisfy

$$
\nu_{1} s^{\alpha} \leq k(s) \leq \nu_{2} s^{\beta}, \quad \text { for } s \in(0,1],
$$

with $|\alpha|<1,|\beta|<1, \nu_{1}, \nu_{2}>0$.

Our method of proof adapts to this model and yields at the limit

$$
\frac{\partial \rho}{\partial t}-\Delta F(\rho)=0
$$


where

$$
F(\rho)=\frac{1}{N} \int_{0}^{\rho} \frac{d s}{k(s)} .
$$

In radiative transfer theory, this limit is known as the "Rosseland approximation". Not only this convergence can be shown in a slightly, more general setting than in [BGPS], but our method of proof is completely different and simply relies upon entropy bounds and the "divcurl" lemma. In particular, our proof works if $k(\rho)=\rho^{\alpha}$ with $|\alpha|<1$.

In conclusion, let us indicate that it is possible to interpret both the finite-velocity models and the above model (6.4) in a single setting. We briefly mention this remark: let $V$ be a bounded set on $\mathbb{R}^{N}$, and let $\mu$ be a probability measure on $V$ satisfying

$$
\begin{aligned}
& \int_{V} v_{k} d \mu=0, \quad \text { for all } 1 \leq k \leq N \\
& \int_{V}(v \cdot \xi)^{2} d \mu>0, \quad \text { for all } \xi \in S^{N-1} .
\end{aligned}
$$

We then look for $u_{\varepsilon}=u_{\varepsilon}(x, v, t) \geq 0$, solution on $\mathbb{R}^{N} \times V \times[0, \infty)$ of

$$
\frac{\partial u}{\partial t}+\frac{1}{\varepsilon} v \cdot \operatorname{grad} u=\frac{1}{\varepsilon^{2}} \rho_{\varepsilon}^{\alpha}\left(\rho_{\varepsilon}-u_{\varepsilon}\right),
$$

where $|\alpha|<1$ and

$$
\rho_{\varepsilon}(x, t)=\int_{V} u_{\varepsilon}(x, v, t) d \mu .
$$

The hydrodynamical limit for this equation is then

$$
\frac{\partial \rho}{\partial t}-\sum_{i, j=1}^{N} a_{i j} \frac{\partial^{2}}{\partial x_{i} \partial x_{j}}\left(\frac{\rho^{1-\alpha}}{1-\alpha}\right)=0
$$

where

$$
a_{i j}=\int_{V} v_{i} v_{j} d \mu
$$

Let us observe that the two (or six in three-dimensions) velocity model we were primarily interested in this paper corresponds to $V=\{+1,-1\}$, with $(1 / 2,1 / 2)$ for the probability measure $\mu$, while the Rosseland approximation corresponds to $V=S^{N-1}$ and $d \mu=d \omega /\left|S^{N-1}\right|$. 
We do not wish to give a detailed proof of the above claims about Rosseland approximation or (6.8)-(6.9), since it is a straightforward adaptation of our method of proof. In particular, using the ideas of Section 4, one obtains all the a priori estimates concerning the monotonicity for convex functionals of the solution.

However, there is one point that we need to detail. Indeed, since $V$ is no more finite (in general), we cannot estimate from below $\rho_{\varepsilon} / u_{\varepsilon}$, and thus we have to modify a little bit our use of the dissipation of entropy. More precisely, we obtain $L_{t}^{\infty}\left(L^{1} \cap L^{\infty}\right)$ bounds on $u_{\varepsilon}$ (and thus on $\rho_{\varepsilon}$ ) independently of $\varepsilon$, and from the monotonicity of the entropy we obtain for all $T>0$

$$
\int_{0}^{T} d t \int d x \int_{V} d \mu \rho_{\varepsilon}^{\alpha} \frac{\rho_{\varepsilon}-u_{\varepsilon}}{\varepsilon^{2}} \log \frac{\rho_{\varepsilon}}{u_{\varepsilon}} \leq C,
$$

where $C$ denotes various positive constants independent of $\varepsilon$.

Hence, if we denote by $j_{\varepsilon}$ the flux

$$
j_{\varepsilon}(x, t)=\int_{V} v \frac{u_{\varepsilon}}{\varepsilon} d \mu=\int_{V} v \frac{u_{\varepsilon}-\rho_{\varepsilon}}{\varepsilon} d \mu
$$

we have

$$
\begin{aligned}
\left|j_{\varepsilon}\right|^{2} & \leq\left(\int_{V}|v|\left|\frac{\sqrt{u_{\varepsilon}}-\sqrt{\rho_{\varepsilon}}}{\varepsilon}\right|\left|\sqrt{u_{\varepsilon}}+\sqrt{\rho_{\varepsilon}}\right| d \mu\right)^{2} \\
& \leq C \int_{V} \frac{\left(\sqrt{u_{\varepsilon}}-\sqrt{\rho_{\varepsilon}}\right)^{2}}{\varepsilon^{2}} d \mu \int_{V}\left(u_{\varepsilon}+\rho_{\varepsilon}\right) d \mu \\
& \leq C \rho_{\varepsilon} \int_{V} \frac{\rho_{\varepsilon}-u_{\varepsilon}}{\varepsilon^{2}} \log \frac{\rho_{\varepsilon}}{u_{\varepsilon}} d \mu,
\end{aligned}
$$

where we used the classical inequality, valid for all $a, b \geq 0$

$$
(\sqrt{a}-\sqrt{b})^{2} \leq C(a-b) \log \frac{a}{b} .
$$

In particular, we deduce from (6.10) that $\left|j_{\varepsilon}\right|^{2} \rho_{\varepsilon}^{\alpha-1}$ is bounded in $L^{1}$ and thus, since $|\alpha|<1, j_{\varepsilon}$ is bounded in $L^{2}$.

The convergence analysis follows along the same lines as in the preceding sections, writing the macroscopic equations for the equation $(6.4)$

$$
\left\{\begin{array}{l}
\frac{\partial \rho_{\varepsilon}}{\partial t}+\operatorname{div} j_{\varepsilon}=0 \\
\varepsilon^{2} \frac{\partial j_{\varepsilon}}{\partial t}+\operatorname{div} \int_{V} v \otimes v u_{\varepsilon} d \mu=-\rho_{\varepsilon}^{\alpha} j_{\varepsilon}
\end{array}\right.
$$


observing that $j_{\varepsilon}, \rho_{\varepsilon}^{\alpha} j_{\varepsilon}$ are bounded in $L^{2}$, and finally that

$$
\int_{V} v \otimes v u_{\varepsilon} d \mu=A \rho_{\varepsilon}+\int_{V} v \otimes v\left(u_{\varepsilon}-\rho_{\varepsilon}\right) d \mu
$$

from which we deduce that $A \operatorname{grad} \rho_{\varepsilon}$ and thus $\operatorname{grad} \rho_{\varepsilon}$, since by construction the matrix $A$ is positive definite, lie in a compact set of $H_{x, t}^{-1}$. In addition, the integral on the right-hand side converges converges to zero in $L_{x, t}^{2}$ as a consequence of the fact that $j_{\varepsilon}$ is bounded in $L^{2}$, while $V$ is bounded.

Several relevant differences between this approach and the approach by C. Bardos, F. Golse, B. Perthame and R. Sentis [BGPS] are worth emphasizing. First of all, the above proof seems a bit simpler and yields, on the technical side, apparently more general results. But in addition, and this is more important, the compactness phenomena are somewhat different, since velocity averaging (as in [BGPS]) would require the measure $\mu$ to satisfy the condition

$$
\mu\{v \in V: v \cdot \xi=0\}=0, \quad \text { for all } \xi \in S^{N-1},
$$

while we only need that the measure $\mu$ satisfies the second condition in (6.7), namely

$$
\int_{V}(v \cdot \xi)^{2} d \mu>0, \quad \text { for all } \xi \in S^{N-1} .
$$

Acknowledgment. This work was begun while the first author was visiting the University of Pavia for the opening lecture of the Ph.D. courses of the Faculty of Sciences. G. Toscani acknowledges the support of the National Council for Researches of Italy (CNR), Project "Kinetic Equations and Diffusion Problems".

\section{References.}

[Aro] Aronson, D. G., The porous media equations. In Non Linear Diffusion problems. Lecture Notes in Math. 1224, A. Fasano and M. Primicerio Eds., Springer Verlag, 1986.

[BGPS] Bardos, C., Golse, F., Perthame, B., Sentis, R., The nonaccretive radiative transfer equations: existence of solutions and Rosseland approximation. J. Funct. Anal. 77 (1988), 434-460. 
[Car] Carleman, T., Problèmes Matematiques dans la Théorie Cinétique des Gaz. Almqvist-Wiksells, 1957.

[Fi1] Fitzgibbon, W. F., The fluid-dynamical limit of the Carleman equation with reflecting boundary. J. Nonlinear Anal. Theory Meth. Appl. 6 (1982), 695-702.

[Fi2] Fitzgibbon, W. F., Initial boundary value problem for the Carleman equation. Comput. Math. Appl. 9 (1983), 519-525.

[Gol] Goldstein, S., On diffusion by discontinuous movements, and on the telegraph equation. Quart. J. Mech. Appl. Math. 4 (1951), 129-156.

[HePi] Herrero, M. A., Pierre, M., The Cauchy Problem for $u_{t}=\Delta u^{m}$ when $0<m<1$. Trans. Amer. Math. Soc. 291 (1985), 145-158.

[Ino] Inoue, M., Derivation of a porous medium equation from many Markovian particles and the propagation of chaos. Hiroshima Math. J. 21 (1991), 85-110.

[KLR] Kaper, H., Leaf, G. K., Reich, S., Convergence of semigroups with an application to the Carleman equation. Math. Meth. Appl. Sci. 2 (1980), 303-308.

[Kur] Kurtz, T. G., Convergence of sequences of semigroups of nonlinear operators with an application to gas kinetics. Trans. Amer. Math. Soc. 186 (1973), 259-272.

[Lio] Lions, J. L., Perturbations singulieres dans les problèmes aux limites et en controle optimale. Lecture Notes in Math. 323. Springer-Verlag, 1973.

[MaMi] Marcati, P., Milani, A. J., The one-dimensional darcy's law as the limit of a compressible euler flow. J. Differential Equations 84 (1990), 129147.

[McK] McKean, H. P., The central limit theorem for Carleman's equation. Israel J. Math. 21 (1975), 54-92.

[Mu1] Murat, F., Compacitè par compensation. Ann. Scuola Norm. Sup. Pisa 5 (1978), 489-507.

[Mu2] Murat, F., Compacitè par compensation II. In Proceedings of the international Meeting on recent methods in Nonlinear Analysis, Roma, May 8-12 1978. E. DeGiorgi, E. Magenes and U. Mosco Eds. Pitagora Editrice, 1979, 245-256.

[Oel] Oelschläger, K., Large systems of interacting particles and the porous medium equation. J. Differential Equations 88 (1990), 294-346.

[PTo] Pulvirenti, A., Toscani, G., Fast diffusion as a limit of a two-velocity kinetic model. Rendiconti del Circolo Matematico di Palermo 45 (1996), 417-426. 
[Ta1] Tartar, L., Homogénéisation et compacité par compensation. Séminaire Goulaouic-Schwartz 1978-79. Ecole Politechnique, Palaiseau (1979) Exposé n. 9 .

[Ta2] Tartar, L., Compensated compactness and applications to partial differential equations. In Non-linear Analysis and Mechanics, Heriot-Watt Symposium, Vol. IV. Ed. by R.J. Knops. Research Notes in Math. 39, Pitman, 1979, 136-212.

[Tay] Taylor, G. I., Diffusion by continuous movements. Proc. London Math. Soc. 20 (1921), 196-212.

Recibido: 4 de marzo de 1.996

Revisado: 29 de enero de 1.997

Pierre Louis Lions CEREMADE, URA CNRS 749

Université Paris-Dauphine Place de Lattre de Tassigny 75775 Paris Cedex 16, FRANCE

and

Giuseppe Toscani

Department of Mathematics

University of Pavia via Abbiategrasso 209 27100 Pavia, ITALY toscani@dragon.ian.pv.cnr.it 